\title{
Forward, backward and elliptic Harnack inequalities for non-negative solutions to certain singular parabolic partial differential equations
}

\author{
Emmanuele DiBenedetto, Ugo GianazZa and Vincenzo Vespri
}

\begin{abstract}
Forward, backward and elliptic Harnack inequalities for non-negative solutions of a class of singular, quasi-linear, parabolic equations, are established. These classes of singular equations include the $p$-Laplacean equation and equations of the porous medium type. Key novel points include form of a Harnack estimate backward in time, that has never been observed before, and measure theoretical proofs, as opposed to comparison principles. These Harnack estimates are established in the super-critical range (1.5) below. Such a range is optimal for a Harnack estimate to hold.
\end{abstract}

Mathematics Subject Classification (2010): 35K65 (primary); 35B65, 35B45 (secondary).

\section{Main results}

Let $E$ be an open set in $\mathbb{R}^{N}$ and for $T>0$ let $E_{T}=E \times(0, T]$. Let $u$ be a weak solution

$$
u \in C_{\mathrm{loc}}\left(0, T ; L_{\mathrm{loc}}^{2}(E)\right) \cap L_{\mathrm{loc}}^{p}\left(0, T ; W_{\mathrm{loc}}^{1, p}(E)\right) \quad 1<p<2
$$

of a quasi-linear, singular parabolic equation of the type

$$
u_{t}-\operatorname{div} \mathbf{A}(x, t, u, D u)=B(x, t, u, D u) \quad \text { weakly in } E_{T}
$$

where the functions $\mathbf{A}: E_{T} \times \mathbb{R}^{N+1} \rightarrow \mathbb{R}^{N}$ and $B: E_{T} \times \mathbb{R}^{N+1} \rightarrow \mathbb{R}$ are only assumed to be measurable and subject to the structure conditions

$$
\left\{\begin{array}{l}
\mathbf{A}(x, t, u, D u) \cdot D u \geq C_{o}|D u|^{p}-C^{p} \\
|\mathbf{A}(x, t, u, D u)| \leq C_{1}|D u|^{p-1}+C^{p-1} \\
|B(x, t, u, D u)| \leq C|D u|^{p-1}+C^{p}
\end{array} \quad \text { a.e. in } E_{T}\right.
$$

This work has been partially supported by I.M.A.T.I. - C.N.R. - Pavia. DiBenedetto's work partially supported by National Science Foundation grant DMS-0652385.

Received March 9, 2009; accepted in revised form May 31, 2009. 
where $p \in(1,2)$ and $C_{o}$ and $C_{1}$ are given positive constants, and $C$ is a given nonnegative constant. If $u$ is a weak solution of (1.1)-(1.2), the quasi-linear structure conditions (1.3) are in addition required to preserve the property of sub(super)solutions of the truncations $\pm(u-k)_{ \pm}$, for all $k \in \mathbb{R}$, where

$$
(u-k)_{+} \equiv \max \{(u-k), 0\}, \quad(u-k)_{-} \equiv \max \{-(u-k), 0\} .
$$

Namely

$$
\frac{\partial}{\partial t}(u-k)_{ \pm}-\operatorname{div} \mathbf{A}\left(x, t,(u-k)_{ \pm}, D(u-k)_{ \pm}\right) \leq B\left(x, t,(u-k)_{ \pm}, D(u-k)_{ \pm}\right)(1.2)_{ \pm}
$$

weakly in $E_{T}$ against admissible non-negative test functions. The prototype example is

$$
u_{t}-\operatorname{div}|D u|^{p-2} D u=0, \quad 1<p<2, \quad \text { weakly in } E_{T} .
$$

This p.d.e. is singular since the modulus of ellipticity $|D u|^{p-2}$ goes to $\infty$ as $|D u| \rightarrow 0$. We will establish that non-negative weak solutions of (1.1)-(1.2) satisfy an intrinsic form of the Harnack inequality provided $p$ is in the super critical range

$$
p_{*}=\frac{2 N}{N+1}<p<2
$$

The parameters $\left\{N, p, C_{o}, C_{1}, C\right\}$ are the data, and we say that a generic constant $\gamma=\gamma\left(N, p, C_{o}, C_{1}, C\right)$ depends upon the data, if it can be quantitatively determined a priori only in terms of the indicated parameters. For $\rho>0$ let $K_{\rho}$ be the cube of center the origin on $\mathbb{R}^{N}$ and edge $2 \rho$ and for $y \in \mathbb{R}^{N}$ let $K_{\rho}(y)$ denote the homothetic cube centered at $y$. Fix $P_{o}=\left(x_{o}, t_{o}\right) \in E_{T}$, such that $u\left(x_{o}, t_{o}\right)>0$, and consider cylinders of the type

$$
Q_{\rho}\left(P_{o}\right)=K_{\rho}\left(x_{o}\right) \times\left\{t_{o}-\left(\frac{u\left(P_{o}\right)}{c^{4}}\right)^{2-p} \rho^{p}<t \leq t_{o}+\left(\frac{u\left(P_{o}\right)}{c^{4}}\right)^{2-p} \rho^{p}\right\},
$$

where $c$ is the constant of Theorem 1.1. These cylinders are "intrinsic" to the solution since their time length is determined by the value of $u$ at $\left(x_{o}, t_{o}\right)$. Cylindrical domains of the form $K_{\rho} \times\left(0, \rho^{p}\right]$, reflect the natural, parabolic space-time dilations that leave (1.4) invariant. The latter however is not homogeneous with respect to the solution $u$. The time dilation by a factor $\left[u\left(P_{o}\right)\right]^{2-p}$ is intended to restore the homogeneity. Then the Harnack inequality holds in such an intrinsic geometry.

Theorem 1.1. Let $u$ be a non-negative, weak solution to (1.1)-(1.3) for $p$ in the super-critical range (1.5). There exist positive constants $\delta_{*}$ and c, depending only upon the data, such that for all $P_{o} \in E_{T}$ and all cylinders of the type $Q_{8 \rho}\left(P_{o}\right) \subset$ $E_{T}$, either $u\left(P_{o}\right) \leq C \rho$, or

$$
c u\left(x_{o}, t_{o}\right) \leq \inf _{K_{\rho}\left(x_{o}\right)} u(\cdot, t)
$$


for all times

$$
t_{o}-\delta_{*}\left[u\left(P_{o}\right)\right]^{2-p} \rho^{p} \leq t \leq t_{o}+\delta_{*}\left[u\left(P_{o}\right)\right]^{2-p} \rho^{p} .
$$

The constants $c$ and $\delta_{*}$ tend to zero as either $p \rightarrow 2$ or as $p \rightarrow p_{*}$.

This inequality is simultaneously a "forward and backward in time" Harnack estimate as well as a Harnack estimate of elliptic type. Inequalities of this type would be false for non-negative solutions of the heat equation. This is reflected in (1.7)-(1.8), as the constants $c$ and $\delta_{*}$ tend to zero as $p \rightarrow 2$. It turns out that these inequalities lose meaning also as $p$ tends to the critical value $p_{*}$ in (1.5). We comment on each these aspects separately.

\subsection{The forward in time Harnack inequality}

A forward Harnack estimate can be established independently of Theorem 1.1 and it takes the following form.

Theorem 1.2. Let $u$ be a non-negative, weak solution to (1.1)-(1.3) for $p$ in the super-critical range (1.5). There exist positive constants $c_{+}, \delta_{+}$such that for all cylinders

$$
K_{8 \rho}\left(x_{o}\right) \times\left\{t_{o}-\left(\frac{u\left(P_{o}\right)}{c_{+}^{4}}\right)^{2-p}(8 \rho)^{p}<t \leq t_{o}+\left(\frac{u\left(P_{o}\right)}{c_{+}^{4}}\right)^{2-p}(8 \rho)^{p}\right\}
$$

contained in $E_{T}$, either $u\left(P_{o}\right)<C \rho$, or

$$
c_{+} u\left(x_{o}, t_{o}\right) \leq \inf _{K_{\rho}\left(x_{o}\right)} u\left(x, t_{o}+\delta_{+}\left[u\left(P_{o}\right)\right]^{2-p} \rho^{p}\right) .
$$

The constants $c_{+}$and $\delta_{+}$tend to zero as $p \rightarrow p_{*}$ but they are "stable" as $p \rightarrow 2$, in the sense that there exist positive constants $c_{+}(2)$ and $\delta_{+}(2)$, that can be determined a priori only in terms of the data, such that $c_{+}(p), \delta_{+}(p) \rightarrow c_{+}(2), \delta_{+}(2)$ as $p \rightarrow 2$. Thus by formally letting $p \rightarrow 2$ in (1.9) one recovers the classical Moser's Harnack inequality of [11].

A positive waiting time is needed, for a Harnack estimate to hold even for non-negative solutions of the heat equation, as pointed out by a counterexample of Moser ([11]). The novelty of (1.9) is in that such a waiting time is intrinsic to the solution itself. No forward in time Harnack estimate would be possible for nonnegative solutions of (1.1)-(1.3) unless the waiting time is driven by the solution itself. Indeed, weak non-negative solutions of (1.4) in bounded domains, with homogeneous Dirichlet data on $\partial E$ and non-negative initial data $u_{o}$, become extinct, abruptly, in finite time. That is, there exists a time $T$ that can be determined a priori in terms of the data and $u_{o}$, such that for all $x \in E$ ([6, Chapter VII, Section 2])

$$
u(x, t)>0 \quad \text { for } t<T \quad \text { and } \quad u(x, t)=0 \quad \text { for } t>T .
$$

For such a solution, a Harnack estimate with waiting time independent of $u$ would not hold. 


\subsection{The elliptic Harnack inequality}

A consequence of (1.7)-(1.8) is the following elliptic form of the Harnack inequality.

Corollary 1.3. Let $u$ be a non-negative, weak solution to (1.1)-(1.3) for $p$ in the super-critical range (1.5). There exists a positive constant $c$, depending only upon the data, such that for all $P_{o} \in E_{T}$ and all cylinders of the type $Q_{8 \rho}\left(P_{o}\right) \subset E_{T}$, either $u\left(P_{o}\right) \leq C \rho$, or

$$
c u\left(x_{o}, t_{o}\right) \leq \inf _{K_{\rho}\left(x_{o}\right)} u\left(\cdot, t_{o}\right)
$$

The constant $c$ tends to zero as either $p \rightarrow 2$ or as $p \rightarrow p_{*}$.

While unusual, such inequality can be understood by examining the nature of (1.4). As $|D u| \approx 0$, the modulus of ellipticity becomes large and the p.d.e. tends to favour its elliptic component. The inequality (1.11) makes this heuristic argument quantitatively precise. The parabolic component enters in that $u$ is required to exist for a sufficiently large time interval about $t_{o}$.

The inequality is false for non-negative solutions of the heat equations $(p=2)$ and is also false for $1<p \leq p_{*}$, as remarked below.

\subsection{The backward in time Harnack inequality}

Another consequence of (1.7)-(1.8) is a backward Harnack estimate in the following form.

Corollary 1.4. Let $u$ be a non-negative, weak solution to (1.1)-(1.3) for $p$ in the super-critical range (1.5). There exist positive constants $\delta_{*}$ and $c$, depending only upon the data, such that for all $P_{o} \in E_{T}$ and all cylinders of the type $Q_{8 \rho}\left(P_{o}\right) \subset$ $E_{T}$, either $u\left(P_{o}\right) \leq C \rho$, or

$$
c u\left(x_{o}, t_{o}\right) \leq \inf _{K_{\rho}\left(x_{o}\right)} u\left(\cdot, t_{o}-\delta_{*}\left[u\left(P_{o}\right)\right]^{2-p} \rho^{p}\right) .
$$

The constants $c$ and $\delta_{*}$ tend to zero as either $p \rightarrow 2$ or as $p \rightarrow p_{*}$.

While unexpected, this occurrence reflects the tendency of the solution to become extinct in finite time, as indicated in (1.10). Despite the form of the inequality, the time is not reversed. Indeed for (1.12) to hold, the solution $u$ is required to exist in a large time-interval about $t_{o}$. Neverthless this remains the most intriguing aspect of these inequalities and we will comment further on it.

\subsection{On the range (1.5) of $p$}

The range of $p$ in (1.5), is optimal for an intrinsic forward in time Harnack estimate (1.9) to hold. For $1<p<p_{*}$ solutions of the Cauchy problem for (1.4) for nonnegative initial data $u_{o} \in L^{1}\left(\mathbb{R}^{N}\right)$ become extinct, abruptly, after a finite time $T$, 
and the solutions exhibit a behavior similar to (1.10) ([6, Chapter VII, Section 3]). Pick $\left(x_{o}, t_{o}\right) \in \mathbb{R}^{N} \times(0, T)$ where $t_{o}$ is so close to $T$ to satisfy

$$
T-t_{o}<\frac{\delta_{+} c_{+}^{4(2-p)}}{8^{p}} t_{o}
$$

and $\delta_{+}$and $c_{+}$are the constants appearing in (1.9). Now choose $\rho>0$ so large that

$$
\delta_{+}\left[u\left(x_{o}, t_{o}\right)\right]^{2-p} \rho^{p}=T-t_{o} .
$$

For such a choice

$$
K_{8 \rho}\left(x_{o}\right) \times\left\{t_{o}-\left(\frac{u\left(P_{o}\right)}{c_{+}^{4}}\right)^{2-p} 8^{p} \rho^{p}, t_{o}+\left(\frac{u\left(P_{o}\right)}{c_{+}^{4}}\right)^{2-p} 8^{p} \rho^{p}\right\} \subset \mathbb{R}^{N} \times(0, \infty)
$$

however the intrinsic Harnack estimate (1.9) fails. For these solutions, also the elliptic version (1.11) fails, as evidenced by the following explicit weak solution of (1.4)

$u(x, t)=\left(|\lambda|\left(\frac{p}{2-p}\right)^{p-1}\right)^{\frac{1}{2-p}} \frac{(T-t)_{+}^{\frac{1}{2-p}}}{|x|^{\frac{p}{2-p}}}, \quad 1<p<\frac{2 N}{N+2}, \quad \lambda=N(p-2)+p$

Such a $u$ is non-negative, unbounded near $x=0$ for all $t<T$ and finite otherwise. Thus (1.11) fails to hold. For $1<p \leq p_{*}$ the mere notion of weak solution is not sufficient to ensure its local boundedness ( [6, Chapter V, Section 5]). The previous solution of (1.4) is indeed unbounded near $x=0$. However the lack of a Harnack estimate is not due to the possible unboundedness of the solutions. The following example can be constructed relying on similar results for the porous medium equation (see [13]), for $p=\frac{2 N}{N+2}<p_{*}$

$$
u(x, t)=(T-t)_{+}^{\frac{N+2}{4}}\left[a+b|x|^{\frac{2 N}{N-2}}\right]^{-\frac{N}{2}}, \quad N>2,
$$

where $a>0$ and $T$ are arbitrary, and

$$
b=b(N, a)=\frac{N-2}{N^{2}}\left(\frac{N+2}{4 N a}\right)^{\frac{N+2}{N-2}} .
$$

Such a function is non-negative, locally bounded, solves (1.4) weakly in $\mathbb{R}^{N} \times \mathbb{R}$, and it does not satisfy the Harnack estimate, in any of the forward, backward or elliptic forms.

The same occurs for the critical value $p=p_{*}$. Indeed the following explicit solution of (1.4), for $p=p_{*}$

$$
u(x, t)=\left[|x|^{\frac{2 N}{N-1}}+e^{b t}\right]^{-\frac{N-1}{2}}, \quad b=\frac{2 N^{\frac{2 N}{N+1}}}{N-1}, \quad N \geq 2
$$


constructed by adapting a result of [9], is positive, locally bounded in the whole $\mathbb{R}^{N} \times \mathbb{R}$, and it fails to satisfy the Harnack estimate in any one of the forward, backward, or elliptic form. These remarks raise the question of what form, if any, the Harnack estimate might take for $p$ in the sub-critical range $1<p \leq p_{*}$.

\subsection{Novelty and significance}

For non-negative solutions of the prototype, homogeneous equation (1.4), intrinsic Harnack inequalities in the forward form (1.9) and the elliptic form (1.11), were established in a series of contributions ([3-5]), collected and re-organized in [6]. These proofs, one way or another had at their root the application of the maximum principle by comparing, locally, the solutions of (1.4) with either the explicit Barenblatt solution ([6]), or some suitably constructed sub-solution ([4]).

The original proofs of the parabolic Harnack inequality for non-negative solutions of the heat equation, due independently to Hadamard [8] and Pini [14], were based on local comparisons with caloric potentials. The leap forward achieved by Moser ( [10-12]) consists in replacing comparison methods by measure-theoretical arguments. This is precisely one of the key novel points of this contribution, that is, the Harnack inequalities (1.7)-(1.9) are established by entirely measure-theoretical arguments, thereby bypassing any form of comparison principle. These arguments are rather different than the classical iteration techniques of De Giorgi [2] and Moser [11]. For degenerate equations (1.1)-(1.3) a similar result has been recently established in [7], to which we refer for further comments.

A second key novel point is the backward inequality in the form (1.12). The latter has never been observed before, not even for the prototype equation (1.4) and it opens intriguing issue on the local behavior of solutions of such singular equations.

The approach is sufficiently general as to apply, by minor modifications, to non-negative weak solutions of a class of singular parabolic equations, including quasi-linear versions of the singular porous-medium equations. Some of these classes and generalizations are indicated in Section 8

\section{Main components in the proof of Theorem 1.1}

\section{1. $L_{\mathrm{loc}}^{1}-L_{\mathrm{loc}}^{\infty}$ Harnack-type estimates for $\lambda>0$}

Theorem 2.1. Let $u$ be a non-negative, weak solution to (1.1)-(1.3) for $p$ in the super-critical range (1.5). There exists a positive constant $\gamma$ depending only upon the data, such that for all cylinders

$$
K_{2 \rho}(y) \times[s-(t-s), s+(t-s)] \subset E_{T}
$$

either

$$
C \rho>\min \left\{1 ;\left(\frac{t-s}{\rho^{p}}\right)^{\frac{1}{2-p}}\right\}
$$


or

$$
\sup _{K_{\rho}(y) \times[s, t]} u \leq \frac{\gamma}{(t-s)^{\frac{N}{\lambda}}}\left(\inf _{2 s-t<\tau<t} \int_{K_{2 \rho}(y)} u(x, \tau) d x\right)^{\frac{p}{\lambda}}+\gamma\left(\frac{t-s}{\rho^{p}}\right)^{\frac{1}{2-p}}
$$

where

$$
\lambda \stackrel{\text { def }}{=} N(p-2)+p .
$$

Remark 2.2. The range (1.5) corresponds to $\lambda>0$. Starting from $K_{\rho}$, the solution $u$ is required to exist in a larger neighborhood $K_{2 \rho}(y)$ and for times comparably larger and smaller than $s$.

\subsection{Expansion of positivity}

Theorem 2.3. Let $u$ be a non-negative, local, weak solution of (1.1)-(1.3) satisfying

$$
\left|[u(\cdot, t)>M] \cap K_{\rho}(y)\right|>\alpha\left|K_{\rho}\right|
$$

for all times

$$
s-(\theta \delta \rho)^{p} \leq t \leq s \quad \text { where } \quad \theta^{p}=M^{2-p}
$$

for some $M>0$, and some $\alpha$ and $\delta$ in $(0,1)$ and assume that $K_{16 \rho}(y) \times[s-$ $\left.(\theta \delta \rho)^{p}, s\right]$ is contained in $E_{T}$. Then, there exists $\eta \in(0,1)$ that can be determined a priori, quantitatively only in terms of the data, and the numbers $\alpha$ and $\delta$, and is independent of $M$, such that either $C \rho>\min \{1 ; M\}$ or

$$
u(x, t) \geq \eta M \quad \text { for all } x \in K_{2 \rho}(y)
$$

for all times

$$
s-\left(\theta \delta \frac{1}{2} \rho\right)^{p}<t \leq s .
$$

Thus measure theoretical information on the measure of the "positivity set" in $K_{\rho}(y)$ for all times in (2.6) implies that such a positivity set actually expands to $K_{2 \rho}(y)$ for comparable times.

Assuming these theorems for the moment, we proceed to establish the Harnack estimates of Theorem 1.1.

\section{An auxiliary proposition}

Weak solutions of (1.1)-(1.3), for $p$ in the range (1.5), are locally bounded and locally Hölder continuous within their domain of definition. Having fixed $\left(x_{o}, t_{o}\right) \in$ $E_{T}$, let $R$ be the largest positive number satisfying

$$
\sup _{K_{R}\left(x_{o}\right)} u\left(x, t_{o}\right)=\mathcal{M}
$$


and

$$
\mathcal{Q}_{\mathcal{M}}\left(P_{o}\right)=K_{8 R}\left(x_{o}\right) \times\left(t_{o}-\mathcal{M}^{2-p}(8 R)^{p}, t_{o}+\mathcal{M}^{2-p}(8 R)^{p}\right] \subset E_{T} .
$$

The upper bound $\mathcal{M}$ and the number $R$ are only known qualitatively. Assume in addition that

$$
C R \leq \min \left\{1 ; u\left(x_{o}, t_{o}\right)\right\}
$$

where $C$ is the constant in the structure conditions (1.3).

Proposition 3.1. Let $u$ be a non-negative, weak solution to (1.1)-(1.3) for $p$ in the super-critical range (1.5). There exist positive constants $\delta_{*}$ and $c$, depending only upon the data, such that

$$
c u\left(x_{o}, t_{o}\right) \leq \inf _{K_{R}\left(x_{o}\right)} u(\cdot, t)
$$

for all times

$$
t_{o}-\delta_{*}\left[u\left(P_{o}\right)\right]^{2-p} R^{p} \leq t \leq t_{o}+\delta_{*}\left[u\left(P_{o}\right)\right]^{2-p} R^{p} .
$$

The constants $c$ and $\delta_{*}$ tend to zero as either $p \rightarrow 2$ or as $p \rightarrow p_{*}$.

The difference between this inequality and that in Theorem 1.1 is that (3.4)(3.5) is established for the specific radius $R$ for which (3.1)-(3.3) hold. As indicated before the value of $R$ is only qualitatively known. Part of the proof of Theorem 1.1, will be to turn these qualitative information into quantitative ones. Assuming them for the moment, introduce the change of variables and unknown function

$$
x \rightarrow \frac{x-x_{o}}{R}, \quad t \rightarrow \frac{t-t_{o}}{\left[u\left(P_{o}\right)\right]^{2-p} R^{p}}, \quad v=\frac{u}{u\left(P_{o}\right)} .
$$

This maps $\mathcal{Q}_{\mathcal{M}}\left(P_{o}\right)$ into

$$
\mathcal{Q}_{\mathcal{M}}=K_{8} \times\left(-\left(\frac{\mathcal{M}}{u\left(P_{o}\right)}\right)^{2-p} 8^{p},\left(\frac{\mathcal{M}}{u\left(P_{o}\right)}\right)^{2-p} 8^{p}\right] .
$$

Relabeling by $x, t$ the new coordinates, $v$ is a weak solution of

$$
v_{t}-\operatorname{div} \overline{\mathbf{A}}(x, t, v, D v)=\bar{B}(x, t, v, D v)
$$

in $\mathcal{Q}_{\mathcal{M}}$. Taking into account (3.3), the functions $\overline{\mathbf{A}}$ and $\bar{B}$ satisfy the structure conditions

$$
\left\{\begin{array}{l}
\overline{\mathbf{A}}(x, t, v, D v) \cdot D v \geq C_{o}|D v|^{p}-1 \\
|\overline{\mathbf{A}}(x, t, v, D v)| \leq C_{1}|D v|^{p-1}+1 \\
|\bar{B}(x, t, v, D v)| \leq|D v|^{p-1}+1
\end{array}\right.
$$

where $C_{o}$ and $C_{1}$ are the constants appearing in (1.3). Establishing Proposition 3.1, consists in finding positive constants $\delta_{*}$ and $c$, depending only upon the data, such that

$$
v(x, t) \geq c \quad \text { for all } x \in K_{1}, \quad \text { for all } t \in\left[-\delta_{*}, \delta_{*}\right] .
$$

In the following we assume $v$ continuous, only in order to give unambiguous meaning to $\sup v$, but in no way the modulus of continuity of $v$ is used in the proof. 


\section{Locating the supremum of $v$ in $K_{1}$}

For $\tau \in(0,1)$ introduce the family of nested expanding cubes $\left\{K_{\tau}\right\}$ centered at the origin, and the increasing families of positive numbers

$$
M_{\tau}=\sup _{K_{\tau}} v, \quad N_{\tau}=(1-\tau)^{-\beta}
$$

where $\beta$ is a positive parameter to be fixed later. As $\tau \rightarrow 1, N_{\tau} \rightarrow \infty$, whereas $M_{\tau}$ remains finite. Therefore the equation $M_{\tau}=N_{\tau}$ has roots. Denoting by $\tau_{o}$ the largest root

$$
M_{\tau_{o}}=\left(1-\tau_{o}\right)^{-\beta} \quad \text { and } \quad M_{\tau} \leq N_{\tau} \quad \text { for all } \tau \geq \tau_{o} .
$$

Since $v$ is continuous, the supremum $M_{\tau_{o}}$ is achieved at some $\bar{x} \in K_{\tau_{o}}$. Choose $\tau_{1} \in(0,1)$ from

$$
\left(1-\tau_{1}\right)^{-\beta}=4\left(1-\tau_{o}\right)^{-\beta} \quad \text { i.e., } \quad \tau_{1}=1-4^{-\frac{1}{\beta}}\left(1-\tau_{o}\right) .
$$

Set also

$$
2 r \stackrel{\text { def }}{=} \tau_{1}-\tau_{o}=\left(1-4^{-\frac{1}{\beta}}\right)\left(1-\tau_{o}\right) .
$$

For these choices, $K_{2 r}(\bar{x}) \subset K_{\tau_{1}}, M_{\tau_{1}} \leq N_{\tau_{1}}$, and

$$
\begin{aligned}
\sup _{K_{\tau_{o}}} v(\cdot, 0) & =\left(1-\tau_{o}\right)^{-\beta}=v(\bar{x}, 0) \leq \sup _{K_{2 r}(\bar{x})} v(\cdot, 0) \\
& \leq \sup _{K_{\tau_{1}}} v(\cdot, 0) \leq 4\left(1-\tau_{o}\right)^{-\beta} .
\end{aligned}
$$

The information on $\tau_{o}$ is only qualitative. By using the parameter $\beta$ we will eliminate such a qualitative dependence from our arguments. The qualitative information on $\mathcal{M}$ still remains and it will be removed as a final step. Because of this interplay between qualitative and quantitative information, our quantitative arguments below are deviced not to depend upon $\beta, \mathcal{M}$ and $R$.

\section{Estimating the sup of $v$ in some intrinsic neighborhood about $(\bar{x}, 0)$}

Consider the cylinder centered at $(\bar{x}, 0)$

$$
Q_{2 r}=K_{2 r}(\bar{x}) \times\left(-\left(\theta_{o} 2 r\right)^{p},\left(\theta_{o} 2 r\right)^{p}\right] \quad \text { where } \theta_{o}^{p}=\left(1-\tau_{o}\right)^{-\beta(2-p)} .
$$

Such a cylinder is included in the box $\mathcal{Q}_{\mathcal{M}}$ introduced in (3.6) since

$$
\begin{aligned}
\left(\theta_{o} 2 r\right)^{p} & =\left(1-\tau_{o}\right)^{-\beta(2-p)}\left(1-4^{\left.-\frac{1}{\beta}\right)^{p}\left(1-\tau_{o}\right)^{p}}\right. \\
& \leq\left(1-\tau_{o}\right)^{-\beta(2-p)}=\left(\frac{u(\bar{x}, 0)}{u\left(P_{o}\right)}\right)^{2-p} \leq\left(\frac{\mathcal{M}}{u\left(P_{o}\right)}\right)^{2-p} .
\end{aligned}
$$


Remark 5.1. For such an inclusion to hold the number $\mathcal{M}$ should not exceed the supremum of the original $u\left(\cdot, t_{o}\right)$ at the fixed time level $t_{o}$. This justifies the choice of $\mathcal{M}$ in (3.1). Such a $\mathcal{M}$ however, is still qualitatively determined.

Proposition 5.2. There exists a constant $\gamma_{1}=\gamma_{1}$ (data), independent of $\beta, \mathcal{M}$ and $R$, such that

$$
\sup _{Q_{r}} v \leq \gamma_{1}\left(1-\tau_{o}\right)^{-\beta}
$$

The constant $\gamma_{1} \rightarrow \infty$ as $p \rightarrow 2$ and as $p \rightarrow p_{*}$.

Proof. Apply (2.1) of Theorem 2.1 to the function $v$, solution of (3.7)-(3.8), over the pair of cylinders $Q_{r} \subset Q_{2 r}$ two times, first for the choice $s=0, t=\left(\theta_{o} 2 r\right)^{p}$, and then for $s=-\left(\theta_{o} r\right)^{p}, t=0$. Taking into account the structure conditions (3.8), and the definition of $\theta_{o}$, the condition (2.2) is always violated. Therefore Theorem 2.1 with these stipulations, gives

$$
\begin{aligned}
\sup _{Q_{r}} v & \leq \gamma\left(1-\tau_{o}\right)^{-\beta \frac{N(p-2)}{\lambda}}\left(\frac{1}{\left|K_{2 r}\right|} \int_{K_{2 r}(\bar{x})} v(x, 0) d x\right)^{\frac{p}{\lambda}}+\gamma 2^{\frac{p}{2-p}} \theta_{o}^{\frac{p}{2-p}} \\
& \leq \gamma\left(4^{\frac{p}{\lambda}}+2^{\frac{p}{2-p}}\right)\left(1-\tau_{o}\right)^{-\beta}=\gamma_{1}\left(1-\tau_{o}\right)^{-\beta}
\end{aligned}
$$

Introduce next the cylinder

$$
Q_{r}\left(\delta \theta_{o}\right)=K_{r}(\bar{x}) \times\left(-\left(\delta \theta_{o} r\right)^{p},\left(\delta \theta_{o} r\right)^{p}\right] \subset Q_{2 r}
$$

where $\delta \in(0,1)$ is to be chosen.

Proposition 5.3. There exist numbers $\delta, \epsilon$, and $\alpha$ in $(0,1)$, depending only upon the data and independent of $\beta, \mathcal{M}$ and $R$, such that

$$
\begin{aligned}
& \left|\left[v(\cdot, t) \geq \epsilon\left(1-\tau_{o}\right)^{-\beta}\right]\right|>\alpha\left|K_{r}\right| \\
& \text { for all } t \in\left[-\left(\delta \theta_{o} r\right)^{p},\left(\delta \theta_{o} r\right)^{p}\right] \\
& \text { where } \theta_{o}^{p}=\left(1-\tau_{o}\right)^{-\beta(2-p)} .
\end{aligned}
$$

The constants $\delta, \epsilon$ and $\alpha$ tend to zero as either $p \rightarrow 2$ or as $\lambda \rightarrow 0$, i.e., as p tends to the critical value $p_{*}$ in (1.5).

Proof. Apply (2.1) of Theorem 2.1 to the function $v$, solution of (3.7)-(3.8), over the pair of cylinders $Q_{\frac{1}{2} r}\left(\delta \theta_{o}\right) \subset Q_{r}\left(\delta \theta_{o}\right)$, for the choices $s=0, t=\left(\delta \theta_{o} r\right)^{p}$. Taking into account the structure conditions (3.8), and the definition of $\theta_{o}$, the condition (2.2) is always violated. Therefore for all $t \in\left[-\left(\delta \theta_{o} r\right)^{p},\left(\delta \theta_{o} r\right)^{p}\right]$

$$
\begin{aligned}
\left(1-\tau_{o}\right)^{-\beta} & =v(\bar{x}, 0) \leq \sup _{K_{\frac{1}{2} r}(\bar{x})} v(\cdot, 0) \\
& \leq \frac{\gamma\left(1-\tau_{o}\right)^{-\beta\left(1-\frac{p}{\lambda}\right)}}{\delta^{\frac{N p}{\lambda}}}\left(\frac{1}{\left|K_{r}\right|} \int_{K_{r}} v(x, t) d x\right)^{\frac{p}{\lambda}}+\gamma(2 \delta)^{\frac{p}{2-p}}(1-\tau)^{-\beta} .
\end{aligned}
$$


Choose $\delta$ from

$$
\gamma(2 \delta)^{\frac{p}{2-p}} \leq \frac{1}{2} \quad \text { and set } \quad \gamma_{2}=2 \gamma, \quad \gamma_{3}=\frac{2^{\frac{N(2-p)}{\lambda}} \gamma_{2}}{\delta^{\frac{N p}{\lambda}}} .
$$

For such choices, $\delta, \gamma_{2}$ and $\gamma_{3}$ depend only upon the data and are independent of $\beta$. Then for all $t \in\left[-\left(\delta \theta_{o} r\right)^{p},\left(\delta \theta_{o} r\right)^{p}\right]$

$$
\frac{1}{\gamma_{2}}\left(1-\tau_{o}\right)^{-\beta} \leq \frac{\left(1-\tau_{o}\right)^{-\beta \frac{N(p-2)}{\lambda}}}{\delta^{\frac{N p}{\lambda}}}\left(\frac{1}{\left|K_{r}\right|} \int_{K_{r}} v(x, t) d x\right)^{\frac{p}{\lambda}} .
$$

From this for $\epsilon \in(0,1)$

$$
\begin{aligned}
\frac{1}{\gamma_{3}}\left(1-\tau_{o}\right)^{-\beta} \leq & \frac{\left(1-\tau_{o}\right)^{-\beta \frac{N(p-2)}{\lambda}}}{2^{\frac{N(2-p)}{\lambda}}}\left(\frac{1}{\left|K_{r}\right|} \int_{K_{r}} v(x, t) d x\right)^{\frac{p}{\lambda}} \\
\leq & \left(1-\tau_{o}\right)^{-\beta \frac{N(p-2)}{\lambda}}\left(\frac{1}{\left|K_{r}\right|} \int_{K_{r}} v(x, t) \chi_{\left[v(\cdot, t)<\epsilon\left(1-\tau_{o}\right)^{-\beta}\right]} d x\right)^{\frac{p}{\lambda}} \\
& +\left(1-\tau_{o}\right)^{-\beta \frac{N(p-2)}{\lambda}}\left(\frac{1}{\left|K_{r}\right|} \int_{K_{r}} v(x, t) \chi_{\left[v(\cdot, t) \geq \epsilon\left(1-\tau_{o}\right)^{-\beta}\right]} d x\right)^{\frac{p}{\lambda}} \\
\leq & \epsilon^{\frac{p}{\lambda}}\left(1-\tau_{o}\right)^{-\beta}+\gamma_{1}^{\frac{p}{\lambda}}\left(1-\tau_{o}\right)^{-\beta}\left(\frac{1}{\left|K_{r}\right|} \int_{K_{r}} \chi_{\left[v(\cdot, t) \geq \epsilon\left(1-\tau_{o}\right)^{-\beta}\right]} d x\right)^{\frac{p}{\lambda}} .
\end{aligned}
$$

To prove (5.1) choose

$$
\epsilon^{\frac{p}{\lambda}}=\frac{1}{2 \gamma_{3}} \quad \text { and set } \quad \alpha=\frac{1}{\gamma_{1}}\left(\frac{1}{2 \gamma_{3}}\right)^{\frac{\lambda}{p}} .
$$

\section{Expanding the positivity of $v$}

Apply Theorem 2.3 to $v$ with $\rho=r, M=\epsilon\left(1-\tau_{o}\right)^{-\beta}$, with $\theta=\theta_{\epsilon}$ given by

$$
\theta_{\epsilon}^{p}=\left[\epsilon\left(1-\tau_{o}\right)^{-\beta}\right]^{2-p}=\epsilon^{2-p} \theta_{o}^{p}
$$

and with $s$ ranging over

$$
-\left(\delta \theta_{o} r\right)^{p}+\left(\delta \theta_{\epsilon} r\right)^{p}<s<\left(\delta \theta_{o} r\right)^{p} .
$$

It gives

$$
v(x, t)>\eta \epsilon\left(1-\tau_{o}\right)^{-\beta} \quad \text { for all } x \in K_{2 r}(\bar{x})
$$

and for all times

$$
-\left(\delta \theta_{o} r\right)^{p}+\left(\delta \theta_{\epsilon} r\right)^{p}<t<\left(\delta \theta_{o} r\right)^{p} .
$$


Apply again Theorem 2.3 with $\rho=2 r, M=\eta \epsilon(1-\tau)^{-\beta}$ and $\theta=\theta_{\epsilon \eta}$ given by

$$
\theta_{\epsilon \eta}^{p}=\left[\epsilon \eta\left(1-\tau_{o}\right)^{-\beta}\right]^{2-p}=(\epsilon \eta)^{2-p} \theta_{o}^{p}
$$

and for all $s$ in the range

$$
-\left(\delta \theta_{o} r\right)^{p}+\left(\delta \theta_{\epsilon} r\right)^{p}+\left(\delta \theta_{\epsilon \eta} 2 r\right)^{p}<s<\left(\delta \theta_{o} r\right)^{p} .
$$

It gives

$$
v(x, t)>\eta^{2} \epsilon\left(1-\tau_{o}\right)^{-\beta} \quad \text { for all } x \in K_{4 r}
$$

for all times

$$
-\left(\delta \theta_{o} r\right)^{p}+\left(\delta \theta_{\epsilon} r\right)^{p}+\left(\delta \theta_{\epsilon \eta} 2 r\right)^{p}<t<\left(\delta \theta_{o} r\right)^{p}
$$

After $n$ iterations

$$
v(x, t)>\eta^{n} \epsilon\left(1-\tau_{o}\right)^{-\beta} \quad \text { for all } x \in K_{2^{n+1} r}
$$

for all times

$$
-\left(\delta \theta_{o} r\right)^{p}+\sum_{j=0}^{n}\left(\delta \theta_{\epsilon \eta^{j}} 2^{j} r\right)^{p}<t<\left(\delta \theta_{o} r\right)^{p} .
$$

Recall the definition (4.1) of $r$ and choose $n$ so large that

$$
1 \leq 2^{n} r \leq 2 \quad \text { which implies } \quad\left(1-\tau_{o}\right)^{-1}>2^{n-2}\left(1-4^{-\frac{1}{\beta}}\right) .
$$

Since $\tau_{o}$ is only known qualitatively also $n$ is qualitative. We remove such a qualitative dependence for a suitable choice of $\beta$ as follow. Taking into account the lower bound in (6.1) and the previous choice of $n$

$$
\eta^{n} \epsilon\left(1-\tau_{o}\right)^{-\beta}>\epsilon 2^{-2 \beta}\left(1-4^{-\frac{1}{\beta}}\right)^{\beta}\left(\eta 2^{\beta}\right)^{n} .
$$

Choose $\beta$ so large that

$$
\eta 2^{\beta}=1 \quad \text { and set } \quad c=\epsilon 2^{-2 \beta}\left(1-4^{-\frac{1}{\beta}}\right)^{\beta} .
$$

Finally, by choosing $\epsilon$ even smaller if necessary we may insure that

$$
\sum_{j=0}^{\infty}\left(\delta \theta_{\epsilon \eta^{j}} 2^{j} r\right)^{p} \leq \frac{1}{2}\left(\delta \theta_{o} r\right)^{p} .
$$

Thus

$$
v(x, t) \geq c \quad \text { for all } x \in K_{1}
$$

for all times

$$
-\frac{1}{2}\left(\delta \theta_{o} r\right)^{p}<t<\left(\delta \theta_{o} r\right)^{p} .
$$


As indicated earlier the information on $\tau_{o}$ is only qualitative and as such, the range of times in (6.3) is qualitative. However from the definition (4.1) of $r$ and (5.1) of $\theta_{o}$

$$
\begin{aligned}
\frac{1}{2}\left(\delta \theta_{o} r\right)^{p} & =\frac{\delta^{p}}{2^{p+1}}\left(1-4^{-\frac{1}{\beta}}\right)^{p}\left(1-\tau_{o}\right)^{-\beta(2-p)}\left(1-\tau_{o}\right)^{p} \\
& \geq \frac{\delta^{p}}{2^{p+1}}\left(1-4^{-\frac{1}{\beta}}\right)^{p}=\delta_{*}
\end{aligned}
$$

provided $\beta \geq p /(2-p)$, which we may assume by possibly taking $\eta$ smaller if necessary. Thus (6.2) holds for all times $t \in\left(-\delta_{*}, \delta_{*}\right)$ and establishes (3.9).

\section{Removing the qualitative information on $\mathcal{M}$ and $R$}

The constants in (3.4)-(3.5), do not depend upon $\mathcal{M}$ nor $R$. These parameters are qualitatively chosen to insure the inclusion (3.2) and play no further role otherwise. However the inequality (3.4)-(3.5) of Proposition 3.1 holds in the qualitative geometry determined by $R$. We will remove such a qualitative dependence to infer an entirely local and quantitative Harnack estimate as stated in Theorem 1.1. We commence by recording two consequences of Proposition 3.1.

Corollary 7.1. Let $u$ be a non-negative, weak solution to (1.1)-(1.3) for $p$ in the super-critical range (1.5). Fix $\left(x_{o}, t_{o}\right) \in E_{T}$ and let $R$ be the largest positive number such that, setting

$$
\mathcal{M}_{n}=\sup _{K_{2^{n}}\left(x_{o}\right)} u\left(\cdot, t_{o}\right)
$$

there holds the inclusions $K_{8\left(2^{n} R\right)}\left(x_{o}\right) \subset E$, and

$$
\left(t_{o}-\mathcal{M}_{n}^{2-p} 8^{p}\left(2^{n} R\right)^{p}, t_{o}+\mathcal{M}_{n}^{2-p} 8^{p}\left(2^{n} R\right)^{p}\right] \subset(0, T]
$$

Then either $C 2^{n} R>\min \left\{1 ; u\left(x_{o}, t_{o}\right)\right\}$, or

$$
c u\left(x_{o}, t_{o}\right) \leq \inf _{x \in K_{2^{n} R}\left(x_{o}\right)} u(\cdot, t)
$$

for all times

$$
t_{o}-\delta_{*}\left[u\left(x_{o}, t_{o}\right)\right]^{2-p}\left(2^{n} R\right)^{p} \leq t \leq t_{o}+\delta_{*}\left[u\left(x_{o}, t_{o}\right)\right]^{2-p}\left(2^{n} R\right)^{p} .
$$

Corollary 7.2. Let $u$ be a non-negative, weak solution to (1.1)-(1.3) for $p$ in the super-critical range (1.5). Fix $P_{o}=\left(x_{o}, t_{o}\right) \in E_{T}$ and let $R$ be the largest positive number such that $K_{8\left(2^{n+1} R\right)}\left(x_{o}\right) \subset E$, and

$$
\left(t_{o}-\mathcal{M}_{n+1}^{2-p} 8^{p}\left(2^{n} R\right)^{p}, t_{o}+\mathcal{M}_{n+1}^{2-p} 8^{p}\left(2^{n} R\right)^{p}\right] \subset(0, T]
$$


Then for all $y \in K_{2^{n} R}\left(x_{o}\right)$, either $C 2^{n} R>\min \left\{1 ; u\left(y, t_{o}\right)\right\}$, or

$$
c \sup _{K_{2^{n} R}\left(x_{o}\right)} u\left(\cdot, t_{o}\right) \leq u\left(y, t_{o}\right) \leq c^{-1} \inf _{x \in K_{2^{n} R}\left(x_{o}\right)} u(\cdot, t)
$$

for the same constant $c$ as in Proposition 3.1, and at the same time level $t_{0}$.

The constant $c=c$ (data) being determined, one may assume that

$$
u\left(x_{o}, t_{o}\right) \leq c^{4} \mathcal{M}_{o} .
$$

Indeed if not, any radius $\rho>0$ for which

$$
\left(t_{o}-\left(\frac{u\left(P_{o}\right)}{c^{4}}\right)^{2-p}(8 \rho)^{p}, t_{o}+\left(\frac{u\left(P_{o}\right)}{c^{4}}\right)^{2-p}(8 \rho)^{p}\right] \subset(0, T)
$$

would satisfy (3.1)-(3.2) and the conclusion of Theorem 1.1 follows from Proposition 3.1 .

Proposition 7.3. Let $R$ be the largest number for which (7.1)-(7.2) hold for $n=0$, and let (7.5) hold. Then for all $n \in \mathbb{N}$ such that $K_{8\left(2^{n} R\right)}\left(x_{o}\right) \subset E$, and

$$
\left(t_{o}-\left(\frac{u\left(P_{o}\right)}{c^{4}}\right)^{2-p} 8^{p}\left(2^{n} R\right)^{p}, t_{o}+8^{p}\left(2^{n} R\right)^{p}\left(\frac{u\left(P_{o}\right)}{c^{4}}\right)^{2-p}\right] \subset(0, T)
$$

there holds

$$
c u\left(x_{o}, t_{o}\right) \leq \inf _{x \in K_{2^{n} R}\left(x_{o}\right)} u(\cdot, t)
$$

for all times

$$
t_{o}-\delta_{*}\left(\frac{u\left(P_{o}\right)}{c^{4}}\right)^{2-p}\left(2^{n} R\right)^{p} \leq t \leq t_{o}+\delta_{*}\left(\frac{u\left(P_{o}\right)}{c^{4}}\right)^{2-p}\left(2^{n} R\right)^{p} .
$$

The constants $c$ and $\delta_{*}$ are the same as in Proposition 3.1.

Proof. The statement holds for $n=0$. Assuming it holds for $n$ it will be shown by induction that continues to hold for $n+1$. For $y \in K_{2^{n+1} R}\left(x_{o}\right)$ the point $z=x_{o}+\frac{1}{2} y$ is in $K_{2^{n}}{ }\left(x_{o}\right)$ and by the induction hypothesis and Corollary 7.1

$$
c \sup _{K_{2^{n} R}(z)} u(\cdot) \leq u(z) \leq c^{-1} \inf _{K_{2^{n} R}(z)} u(\cdot) .
$$

Since $y \in K_{2^{n+1} R}\left(x_{o}\right)$ is arbitrary, this implies

$$
\mathcal{M}_{n+1}=\sup _{y \in K_{2^{n+1} R}\left(x_{o}\right)} u\left(\cdot, t_{o}\right) \leq c^{-2} u\left(x_{o}, t_{o}\right) .
$$


Moreover, taking into account (7.5)

$$
\mathcal{M}_{n+1}^{2-p} 8^{p}\left(2^{n+1} R\right)^{p} \leq 2^{p} c^{2(2-p)}\left(\frac{u\left(P_{o}\right)}{c^{4}}\right)^{2-p} 8^{p}\left(2^{n} R\right)^{p}
$$

provided $2^{p} c^{2(2-p)} \leq 1$, which we may assume. Thus $\mathcal{M}_{n+1}$ and $2^{n+1} R$ satisfy the assumption of Corollary 7.1, and the conclusion follows from (7.3)-(7.4) for the index $n+1$.

\subsection{Proof of Theorem 1.1 concluded}

Fix $\left(x_{o}, t_{o}\right) \in E_{T}$ and pick a radius $\rho$ for which (7.6) is satisfied. Then qualitatively determine $\mathcal{M}$ and $R$ as in (3.1)-(3.2). There exists a non-negative integer $n$ such that $2^{n} R \leq \rho \leq 2^{n+1} R$.

\section{Miscellaneous results}

\subsection{Hölder continuity of solutions}

Locally bounded weak solutions $u$ of (1.1)-(1.2), with no sign restrictions are locally Hölder continuous. Such a local behavior was established in [6, Chapter IV], along with locally quantitative Hölder estimates (see also [1]).

Working exactly as in [7, Section 10], the forward in time Harnack inequality of Theorem 1.2 can be used to establish locally quantitative Hölder estimates for local, weak solutions $u$ of (1.1)-(1.2), thereby providing an alternative proof to [6], for $p$ in the range (1.5).

\subsection{Proof of Theorem 1.2}

The estimates in the proof of Theorem 1.1 are not stable as $p \rightarrow 2$. Stable estimates for $p \rightarrow 2$ required in the proof of Theorem 1.2 can be derived as in [7] by almost identical arguments. Here we point out the the main difference. By the same change of variables as in Section 3, construct the family of expanding cylinders $Q_{\tau} \equiv$ $\{|x|<\tau\} \times\{-\tau, 0\}$ and the numbers

$$
M_{\tau} \equiv\|v\|_{\infty, Q_{\tau}}, \quad N_{\tau} \equiv(1-\tau)^{-\beta},
$$

where again $\beta$ is a positive number to be chosen. Let $\tau_{o}$ be the largest root of the equation $M_{\tau}=N_{\tau}$, so that

$$
M_{\tau_{o}}=\left(1-\tau_{o}\right)^{-\beta}, \quad M_{\frac{1+\tau_{o}}{2}} \leq 2^{\beta}\left(1-\tau_{o}\right)^{-\beta} .
$$

If $(\bar{x}, \bar{t})$ is a point in $Q_{\tau_{o}}$ where $v$ achieves the value $M_{\tau_{o}}$, we have

$$
v(x, t) \leq 2^{\beta}\left(1-\tau_{o}\right)^{-\beta}, \quad|x-\bar{x}|<\frac{1-\tau_{o}}{2}, \quad \bar{t}-\frac{1-\tau_{o}}{2}<t<\bar{t} .
$$


Now let

$$
R_{o}=\frac{1}{2} 2^{-\beta \frac{2-p}{p}}\left(1-\tau_{o}\right)^{\beta \frac{2-p}{p}}\left(1-\tau_{o}\right)
$$

and consider the cylinder

$$
Q_{o}(\bar{x}, \bar{t}) \equiv\left\{|x-\bar{x}|<R_{o}\right\} \times\left\{\bar{t}-\left[2^{\beta}\left(1-\tau_{o}\right)^{-\beta}\right]^{\frac{2-p}{p}} R_{o}^{p}, \bar{t}\right\} .
$$

From the definitions of $Q_{\tau}$ and $R_{o}$, we have $Q_{o}(\bar{x}, \bar{t}) \subset Q_{\frac{1+\tau_{o}}{2}}$, so that by (8.1),

$$
\|v\|_{\infty, Q_{o}(\bar{x}, \bar{t})} \leq 2^{\beta}\left(1-\tau_{o}\right)^{-\beta} .
$$

Therefore $Q_{o}(\bar{x}, \bar{t})$ has the right size. From here on, the proof works exactly as in [7, Sections 7 and 8], to which we refer.

\subsection{Decay in space and time variables}

Let $u$ be a non-negative weak solution of (1.2), fix $\left(x_{o}, t_{o}\right)$ with $u\left(x_{o}, t_{o}\right)>0$, $(x, t)$ in $E_{T}$, with $t>t_{o}$ and $x \neq x_{o}$, and construct the line through them, and the $p$-paraboloid with vertex at $\left(x_{o}, t_{o}\right)$

$$
y(s)-x_{o}=\frac{x-x_{o}}{t-t_{o}}\left(s-t_{o}\right), \quad s-t_{o}=\left(\delta u\left(x_{o}, t_{o}\right)\right)^{2-p}\left|y-x_{o}\right|^{p}
$$

where $\delta=\delta_{+}^{\frac{1}{2-p}}$, and $\delta_{+}$is the constant appearing in the time delay required by Theorem 1.2. The line and the $p$-paraboloid intersect at $\left(x_{1}, t_{1}\right)$ where

$$
\begin{aligned}
\left|x_{1}-x_{o}\right|^{p-1} & =\left(\frac{1}{\delta u\left(x_{o}, t_{o}\right)}\right)^{2-p} \frac{t-t_{o}}{\left|x-x_{o}\right|}, \\
t_{1}-t_{o} & =\left(\delta u\left(x_{o}, t_{o}\right)\right)^{2-p}\left|x_{1}-x_{o}\right|^{p} .
\end{aligned}
$$

Iteration of this process gives a sequence of points $\left\{\left(x_{j}, t_{j}\right)\right\}$ such that

$$
\begin{aligned}
\left|x_{j+1}-x_{j}\right|^{p-1} & =\left(\frac{1}{\delta u\left(x_{j}, t_{j}\right)}\right)^{2-p} \frac{t-t_{o}}{\left|x-x_{o}\right|} \\
t_{j+1}-t_{j} & =\left(\delta u\left(x_{j}, t_{j}\right)\right)^{2-p}\left|x_{j+1}-x_{j}\right|^{p} .
\end{aligned}
$$

The intrinsic geometry of $\left(x_{j}, t_{j}\right)$ permits to relate $u\left(x_{j+1}, t_{j+1}\right)$ to $u\left(x_{j}, t_{j}\right)$, by means of the Harnack inequality, provided the space-time cylinders relative to each $\left(x_{j}, t_{j}\right)$, and defined in (1.6) are all contained in $E_{T}$. Stipulating that $t_{o}<t_{j} \leq t$, this occurs if

$$
\begin{aligned}
& t_{j}-\frac{8}{\left(c_{+}^{4} \delta\right)^{\frac{2-p}{p}}}\left(t_{j+1}-t_{j}\right) \geq t_{o}-\frac{8}{\left(c_{+}^{4} \delta\right)^{\frac{2-p}{p}}}\left(t-t_{o}\right) \geq 0, \\
& K_{8\left|x-x_{o}\right|}\left(x_{o}\right) \subset E .
\end{aligned}
$$


Therefore if $\left(t-t_{o}\right)<\frac{\left(c_{+}^{4} \delta\right)^{\frac{2-p}{p}}}{8} t_{o}$, the conditions of Theorem 1.2 are verified, and

$$
c_{+} u\left(x_{j}, t_{j}\right) \leq u\left(x_{j+1}, t_{j+1}\right) \quad \text { for } j=0,1, \ldots, n
$$

where $n$ is a positive integer to be chosen. From this, by iteration

$$
u\left(x_{n}, t_{n}\right) \geq c_{+}^{n} u\left(x_{o}, t_{o}\right) .
$$

From (8.2)

$$
\begin{aligned}
\left|x-x_{o}\right| & \geq \sum_{j=0}^{n-1}\left|x_{j+1}-x_{j}\right|=\left(\frac{1}{\delta^{2-p}} \frac{t-t_{o}}{\left|x-x_{o}\right|}\right)^{\frac{1}{p-1}} \sum_{j=0}^{n-1}\left(\frac{1}{u\left(x_{j}, t_{j}\right)}\right)^{\frac{2-p}{p-1}} \\
& \geq\left(\frac{t-t_{o}}{\left|x-x_{o}\right|}\right)^{\frac{1}{p-1}}\left(\frac{1}{\delta u\left(x_{n}, t_{n}\right)}\right)^{\frac{2-p}{p-1}} \sum_{j=0}^{n-1}\left(c_{+}^{\frac{2-p}{p-1}}\right)^{n-j} \\
& =\left(\frac{t-t_{o}}{\left|x-x_{o}\right|}\right)^{\frac{1}{p-1}}\left(\frac{1}{\delta u\left(x_{n}, t_{n}\right)}\right)^{\frac{2-p}{p-1}} \frac{q\left(1-q^{n}\right)}{1-q} \quad \text { where } q=c_{+}^{\frac{2-p}{p-1}} .
\end{aligned}
$$

From this

$$
\left(c_{+}^{\frac{2-p}{p-1}}\right)^{n}+\frac{1-q}{q}\left(\frac{\left[\delta u\left(x_{n}, t_{n}\right)\right]^{2-p}\left|x-x_{o}\right|^{p}}{\left(t-t_{o}\right)}\right)^{\frac{1}{p-1}} \geq 1
$$

Without loss of generality, by a possible further application of the Harnack inequality, and by possibly slightly modifying the constant $c_{+}$if needed, we may assume that $\left(x_{n}, t_{n}\right)=(x, t)$. Combining (8.3) and (8.4) we have proved

Proposition 8.1. Let $u$ be a non-negative solution of (1.2) and let $\left(x_{o}, t_{o}\right) \in E_{T}$ be such that $u\left(x_{o}, t_{o}\right)>0$. Then for all $(x, t)$ in $E_{T}$ with $x \neq x_{o} K_{8\left|x-x_{o}\right|}\left(x_{o}\right) \subset E_{T}$ and $0<t-t_{o}<\frac{\left(c_{+}^{4} \delta\right)^{\frac{2-p}{p}}}{8} t_{o}$

$$
\frac{u(x, t)}{u\left(x_{o}, t_{o}\right)} \geq c_{+}\left[1+\gamma\left(\frac{\left[u\left(x_{o}, t_{o}\right)\right]^{2-p}\left|x-x_{o}\right|^{p}}{\left(t-t_{o}\right)}\right)^{\frac{1}{p-1}}\right]^{\frac{p-1}{p-2}}
$$

where

$$
\gamma=\left(c_{+}^{\frac{p-2}{p-1}}-1\right) \delta_{+}^{\frac{1}{p-1}}
$$

Notice that (8.5) gives the same space-decay of the explicit Barenblatt "fundamental solution" of (1.4). As for the decay in the time variable, consider the sequence

$$
t_{o}=s>0, t_{1}=2 s, \ldots, t_{k}=2^{k} s=\tau .
$$


A repeated application of the forward Harnack inequality (1.9) for sufficiently large $s$ yields

$$
u(0, \tau) \geq\left(\frac{s}{\tau}\right)^{a} u(0, s), \quad a=\left|\log _{2} c_{+}\right|
$$

\subsection{Equations of porous medium type}

Consider quasi-linear, singular, parabolic differential equations of the form

$$
u_{t}-\operatorname{div} \mathbf{A}(x, t, u, D u)=B(x, t, u, D u) \quad \text { weakly in } E_{T}
$$

where the functions A : $E_{T} \times \mathbb{R}^{N+1} \rightarrow \mathbb{R}^{N}$ and $B: E_{T} \times \mathbb{R}^{N+1} \rightarrow \mathbb{R}$ are only assumed to be measurable and subject to the structure conditions

$$
\left\{\begin{array}{l}
\mathbf{A}(x, t, u, D u) \cdot D u \geq C_{o}|u|^{m-1}|D u|^{2}-C^{2} \\
|\mathbf{A}(x, t, u, D u)| \leq C_{1}|u|^{m-1}|D u|+C \\
|B(x, t, u, D u)| \leq C|u|^{m-1}|D u|+C^{2}
\end{array}\right.
$$

where $0<m<1$ and $C_{o}$ and $C_{1}$ are given positive constants, and $C$ is a given non-negative constant. If $u$ is a weak solution of (8.7), the quasi-linear structure conditions (8.8) are in addition required to preserve the property of sub(super)solutions of the truncations $\pm(u-k)_{ \pm}$, for all $k \in \mathbb{R}$. Namely

$$
\frac{\partial}{\partial t}(u-k)_{ \pm}-\operatorname{div} \mathbf{A}\left(x, t,(u-k)_{ \pm}, D(u-k)_{ \pm}\right) \leq B\left(x, t,(u-k)_{ \pm}, D(u-k)_{ \pm}\right)
$$

weakly in $E_{T}$ against admissible non-negative test functions. The prototype example is

$$
u_{t}-\Delta\left(|u|^{m-1} u\right)=0, \quad 0<m<1, \quad \text { weakly in } E_{T} .
$$

This p.d.e. is singular since the modulus of ellipticity $m|u|^{m-1}$ goes to $\infty$ as $|u| \rightarrow 0$. Non-negative weak solutions of (8.7)-(8.8) satisfy an intrinsic form of the Harnack inequality provided $m$ is in the super-critical range

$$
m_{*}=\frac{(N-2)_{+}}{N}<m<1
$$

Fix $P_{o}=\left(x_{o}, t_{o}\right) \in E_{T}$, such that $u\left(x_{o}, t_{o}\right)>0$, and consider cylinders of the type

$$
Q_{\rho}\left(P_{o}\right)=K_{\rho}\left(x_{o}\right) \times\left\{t_{o}-\left(\frac{u\left(P_{o}\right)}{\bar{c}^{4}}\right)^{1-m} \rho^{2}<t \leq t_{o}+\left(\frac{u\left(P_{o}\right)}{\bar{c}^{4}}\right)^{1-m} \rho^{2}\right\}
$$

where $\bar{c}$ is the constant of Theorem 8.2. These cylinders are "intrinsic" to the solution since their time length is determined by the value of $u$ at $\left(x_{o}, t_{o}\right)$. 
Theorem 8.2. Let $u$ be a non-negative, weak solution to (8.7)-(8.8) for $m$ in the super-critical range (8.10). There exist positive constants $\bar{\delta}_{*}$ and $\bar{c}$, depending only upon the data, such that for all $P_{o} \in E_{T}$ and all cylinders of the type $Q_{8 \rho}\left(P_{o}\right) \subset$ $E_{T}$, either $u\left(P_{o}\right) \leq C \rho$, or

$$
\bar{c} u\left(x_{o}, t_{o}\right) \leq \inf _{K_{\rho}\left(x_{o}\right)} u(\cdot, t)
$$

for all times

$$
t_{o}-\bar{\delta}_{*}\left[u\left(P_{o}\right)\right]^{1-m} \rho^{2} \leq t \leq t_{o}+\bar{\delta}_{*}\left[u\left(P_{o}\right)\right]^{1-m} \rho^{2} .
$$

The constants $\bar{c}$ and $\bar{\delta}_{*}$ tend to zero as either $m \rightarrow 1$ or as $m \rightarrow m_{*}$.

The same methods leading to the Harnack estimates of Theorem 8.2 can be used to establish the local Hölder continuity of locally bounded weak solutions.

The constants $\bar{c}$ ans $\bar{\delta}_{*}$ can be stabilized in the forward in time Harnack inequality as $m \rightarrow 1$, exactly as for the corresponding quantities for equations (1.1)-(1.3), when $p \rightarrow 2$.

If $\mathbf{A}$ and $B$ are locally analytic in $E_{T} \times \mathbb{R}^{N+1}$, then non-negative weak solutions are locally analytic in the space variables and at least Lipschitz continuous in time.

More precisely, fix $P_{o} \equiv\left(x_{o}, t_{o}\right) \in E_{T}$, such that $u\left(x_{o}, t_{o}\right)>0$, and suppose that $Q_{8 \rho}\left(P_{o}\right) \subset E_{T}$ : working as in [5], the following result can be established:

Theorem 8.3. Let $u$ be a non-negative, weak solution to (8.7)-(8.8) for $m$ in the super-critical range (8.10), with $C=0$, and suppose that $\mathbf{A}$ is locally analytic in $E_{T} \times \mathbb{R}^{N+1}$. Assume moreover that $\mathbf{A}$, whenever well defined, is a locally analytic function of its arguments. Then there exists a positive constant $\gamma$ depending only upon the data and independent of $u$, such that for every multiindex $\alpha$

$$
\left|D^{\alpha} u\left(x_{o}, t_{o}\right)\right| \leq \frac{\gamma^{|\alpha|+1}|\alpha| !}{\rho^{|\alpha|}} u\left(x_{o}, t_{o}\right) .
$$

Moreover for every non-negative integer $k$,

$$
\left|\frac{\partial^{k}}{\partial t^{k}} u\left(x_{o}, t_{o}\right)\right| \leq \frac{\gamma^{2 k+1}(k !)^{2}}{\rho^{2 k}}\left[u\left(x_{o}, t_{o}\right)\right]^{1-(1-m) k} .
$$

By a straightforward approximation procedure, the conclusion of Theorem 8.3 continues to hold for points $\left(x_{o}, t_{o}\right) \in E_{T}$ such that $u\left(x_{o}, t_{o}\right)=0$ (for further details, see [5, Sections 2 and 3]).

\section{Appendices}

\section{A. Proof of Theorem 2.1}

\section{A.1. An $L_{\mathrm{loc}}^{1}$-form of the Harnack inequality for all $1<p<2$}

Proposition A.1. Let u be a non-negative, weak solution to (1.1)-(1.3) for $1<$ $p<2$. There exists a positive constant $\gamma$ depending only upon the data, such that 
for all cylinders $K_{2 \rho}(y) \times[s, t] \subset E_{T}$, either

$$
C \rho>\min \{1 ; \epsilon\} \quad \text { where } \epsilon=\left(\frac{t-s}{\rho^{p}}\right)^{\frac{1}{2-p}}
$$

or

$$
\sup _{s<\tau<t} \int_{K_{\rho}(y)} u(x, \tau) d x \leq \gamma \inf _{s<\tau<t} \int_{K_{2 \rho}(y)} u(x, \tau) d x+\gamma\left(\frac{t-s}{\rho^{\lambda}}\right)^{\frac{1}{2-p}}
$$

where $\lambda$ is defined in (2.4).

The range (1.5) corresponds to $\lambda>0$, and viceversa. However (A.2) holds true for all $1<p<2$ and accordingly, $\lambda$ could be of either sign. The constant $\gamma=\gamma(p) \rightarrow \infty$ as either $p \rightarrow 2$ or as $p \rightarrow 1$.

The theorem was established in [6, Chapter VII, Section 4], for non-negative weak solutions of the prototype homogeneous equation (1.4). Here we give a proof that includes equations of the type of (1.1)-(1.2), with the full quasi-linear structure (1.3).

\section{A.2. An auxiliary lemma}

Lemma A.2. Let $u$ be a non-negative, weak solution to (1.1)-(1.3) for $1<p<2$. There exists a positive constant $\gamma$ depending only upon the data, such that for all cylinders $K_{\rho}(y) \times[s, t] \subset E_{T}$, and all $\sigma \in(0,1)$, and all $\epsilon>0$, either (A.1) holds, or

$$
\begin{aligned}
& \int_{s}^{t} \int_{K_{\sigma \rho}(y)} \tau^{\frac{1}{p}}(u+\epsilon)^{-\frac{2}{p}}|D u|^{p} \zeta^{p} d x d \tau \\
& \leq \frac{\gamma \rho}{(1-\sigma)^{p}}\left(\frac{t-s}{\rho^{\lambda}}\right)^{\frac{1}{p}}\left(\mathcal{S}+\epsilon \rho^{N}\right)^{2 \frac{p-1}{p}}
\end{aligned}
$$

where

$$
\mathcal{S}=\sup _{s<\tau<t} \int_{K_{\rho}(y)} u(x, \tau) d \tau .
$$

The constant $\gamma(p) \rightarrow \infty$ as either $p \rightarrow 1,2$.

Proof. Assume $(y, s)=(0,0)$, fix $\sigma \in(0,1)$ and let $x \rightarrow \zeta(x)$ be a non-negative piecewise smooth cutoff function in $K_{\rho}$ that equals one on $K_{\sigma \rho}$ and such that $|D \zeta| \leq 1 /(1-\sigma) \rho^{p}$. In the weak formulation of (1.2) take the test function

$$
\varphi=-t^{\frac{1}{p}}(u+\epsilon)^{1-\frac{2}{p}} \zeta^{p} \quad \text { for some } \epsilon>0
$$


modulo a Steklov averaging process. This gives

$$
\begin{aligned}
& \frac{2-p}{p} C_{o} \int_{0}^{t} \int_{K_{\rho}} \tau^{\frac{1}{p}}(u+\epsilon)^{-\frac{2}{p}}|D u|^{p} \zeta^{p} d x d \tau \\
& \leq \frac{p}{2(p-1)} t^{\frac{1}{p}} \int_{K_{\rho}}(u+\epsilon)^{2 \frac{p-1}{p}}(x, t) \zeta^{p} d x \\
& \quad+p C_{1} \int_{0}^{t} \int_{K_{\rho}} \tau^{\frac{1}{p}}(u+\epsilon)^{1-\frac{2}{p}}|D u|^{p-1} \zeta^{p-1}|D \zeta| d x d \tau \\
& \quad+\frac{2-p}{p} C^{p} \int_{0}^{t} \int_{K_{\rho}} \tau^{\frac{1}{p}}(u+\epsilon)^{-\frac{2}{p}} \zeta^{p} d x d \tau \\
& \quad+C^{p} \int_{0}^{t} \int_{K_{\rho}}^{\tau^{\frac{1}{p}}(u+\epsilon)^{1-\frac{2}{p}} \zeta^{p} d x d \tau} \\
& \quad+p C^{p-1} \int_{0}^{t} \int_{K_{\rho}} \tau^{\frac{1}{p}}(u+\epsilon)^{1-\frac{2}{p}} \zeta^{p-1}|D \zeta| d x d \tau \\
& \quad+C \int_{0}^{t} \int_{K_{\rho}}^{\tau^{\frac{1}{p}}(u+\epsilon)^{1-\frac{2}{p}}|D u|^{p-1} \zeta^{p} d x d \tau}
\end{aligned}
$$

From this, by repeated application of Young's inequality

$$
\begin{aligned}
& \int_{0}^{t} \int_{K_{\rho}} \tau^{\frac{1}{p}}(u+\epsilon)^{-\frac{2}{p}}|D u|^{p} \zeta^{p} d x d \tau \leq \gamma t^{\frac{1}{p}} \int_{K_{\rho}}(u+\epsilon)^{2 \frac{p-1}{p}}(x, t) \zeta^{p} d x \\
& +\gamma \int_{0}^{t} \int_{K_{\rho}} \tau^{\frac{1}{p}}(u+\epsilon)^{p-\frac{2}{p}}\left(|D \zeta|^{p}+C^{p} \zeta^{p}\right) d x d \tau \\
& +\gamma C^{p} \int_{0}^{t} \int_{K_{\rho}} \tau^{\frac{1}{p}}(u+\epsilon)^{-\frac{2}{p}} \zeta^{p} d x d \tau
\end{aligned}
$$

where $\gamma=\gamma$ (data) tends to $\infty$ as either $p \rightarrow 2$ or as $p \rightarrow 1$. By Hölder's inequality

$$
\begin{aligned}
& \gamma t^{\frac{1}{p}} \int_{K_{\rho}}(u+\epsilon)^{2 \frac{p-1}{p}}(x, t) \zeta^{p} d x \\
& \leq \gamma t^{\frac{1}{p}} \rho^{\frac{N(2-p)}{p}}\left(\sup _{0 \leq \tau \leq t} \int_{K_{\rho}} u(x, \tau) d x+\epsilon(2 \rho)^{N}\right)^{2 \frac{p-1}{p}} \\
& \leq \gamma \rho\left(\frac{t}{\rho^{\lambda}}\right)^{\frac{1}{p}}\left(\mathcal{S}+\epsilon \rho^{N}\right)^{2 \frac{p-1}{p}} .
\end{aligned}
$$


Next

$$
\begin{aligned}
& \gamma \int_{0}^{t} \int_{K_{\rho}} \tau^{\frac{1}{p}}(u+\epsilon)^{p-\frac{2}{p}}\left(|D \zeta|^{p}+C^{p} \zeta^{p}\right) d x d \tau \\
& \leq \gamma \frac{1+C^{p} \rho^{p}}{(1-\sigma)^{p} \rho^{p}} \int_{0}^{t} \int_{K_{\rho}} \tau^{\frac{1}{p}}(u+\epsilon)^{p-2}(u+\epsilon)^{2 \frac{p-1}{p}} d x d \tau \\
& \leq \gamma \frac{1+C^{p} \rho^{p}}{(1-\sigma)^{p}}\left(\frac{t}{\rho^{p}}\right) \epsilon^{p-2} t^{\frac{1}{p}} \sup _{0 \leq \tau \leq t} \int_{K_{\rho}}(u+\epsilon)^{2 \frac{p-1}{p}} d x \\
& \leq \gamma \rho \frac{1+C^{p} \rho^{p}}{(1-\sigma)^{p}}\left(\frac{t}{\rho^{p}}\right) \epsilon^{p-2}\left(\frac{t}{\rho^{\lambda}}\right)^{\frac{1}{p}}\left(\mathcal{S}+\epsilon \rho^{N}\right)^{2 \frac{p-1}{p}} .
\end{aligned}
$$

Finally

$$
\begin{aligned}
& \gamma C^{p} \int_{0}^{t} \int_{K_{\rho}} \tau^{\frac{1}{p}}(u+\epsilon)^{-\frac{2}{p}} \zeta^{p} d x d \tau \\
& \leq \gamma \rho\left(\frac{C \rho}{\epsilon}\right)^{p}\left(\frac{t}{\rho^{p}}\right) \epsilon^{p-2}\left(\frac{t}{\rho^{\lambda}}\right)^{\frac{1}{p}}\left(\mathcal{S}+\epsilon \rho^{N}\right)^{2 \frac{p-1}{p}}
\end{aligned}
$$

Combining these estimates

$$
\begin{aligned}
& \int_{0}^{t} \int_{K_{\rho}} \tau^{\frac{1}{p}}(u+\epsilon)^{-\frac{2}{p}}|D u|^{p} \zeta^{p} d x d \tau \\
& \leq \frac{\gamma \rho}{(1-\sigma)^{p}}\left\{1+\left[1+(C \rho)^{p}+\frac{(C \rho)^{p}}{\epsilon^{p}}\right]\left(\frac{t}{\rho^{p}}\right) \epsilon^{p-2}\right\}\left(\frac{t}{\rho^{\lambda}}\right)^{\frac{1}{p}}\left(\mathcal{S}+\epsilon \rho^{N}\right)^{2 \frac{p-1}{p}} .
\end{aligned}
$$

To prove the Lemma A.2, choose $\epsilon$ as in (A.1) and stipulate that $C$ violates the first of (A.1).

Lemma A.3. Let $u$ be a non-negative, weak solution to (1.1)-(1.3) for $1<p<2$. There exists a positive constant $\gamma$ depending only upon the data, such that for all cylinders $K_{\rho}(y) \times[s, t] \subset E_{T}$, and all $\sigma \in(0,1)$, either (A.1) holds, or

$$
\frac{1}{\rho} \int_{s}^{t} \int_{K_{\sigma \rho}(y)}|D u|^{p-1} d x d \tau \leq \delta \mathcal{S}+\frac{\gamma(p)}{\left[\delta^{2}(1-\sigma)^{p}\right]^{\frac{p-1}{2-p}}}\left(\frac{t-s}{\rho^{\lambda}}\right)^{\frac{1}{2-p}}
$$

for all $\delta \in(0,1)$. The constant $\gamma(p) \rightarrow \infty$ as either $p \rightarrow 1,2$. 
Proof. Continue to assume that $(y, s)=(0,0)$ and that $C$ violates (A.1). By Hölder's and Young's inequalities

$$
\begin{aligned}
& \int_{0}^{t} \int_{K_{\sigma \rho}}|D u|^{p-1} d x d \tau \\
& =\int_{0}^{t} \int_{K_{\sigma \rho}}\left[\tau^{\frac{1}{p} \frac{p-1}{p}}(u+\epsilon)^{-\frac{2}{p} \frac{p-1}{p}}|D u|^{p-1}\right]\left[\tau^{-\frac{1}{p} \frac{p-1}{p}}(u+\epsilon)^{\frac{2}{p} \frac{p-1}{p}}\right] d x d \tau \\
& \leq\left(\int_{0}^{t} \int_{K_{\sigma \rho}} \tau^{\frac{1}{p}}(u+\epsilon)^{-\frac{2}{p}}|D u|^{p} d x d \tau\right)^{\frac{p-1}{p}}\left(\int_{0}^{t} \int_{K_{\sigma \rho}} \tau^{\frac{1}{p}-1}(u+\epsilon)^{2 \frac{p-1}{p}} d x d \tau\right)^{\frac{1}{p}} \\
& \leq \frac{\gamma \rho}{(1-\sigma)^{p-1}}\left(\frac{t}{\rho^{\lambda}}\right)^{\frac{1}{p}}\left(\mathcal{S}+\epsilon \rho^{N}\right)^{2 \frac{p-1}{p}} \leq \delta \rho \mathcal{S}+\frac{\gamma \rho}{\delta^{\frac{2(p-1)}{2-p}}(1-\sigma)^{\frac{p(p-1)}{2-p}}\left(\frac{t}{\rho^{\lambda}}\right)^{\frac{1}{2-p}} \cdot}
\end{aligned}
$$

\section{A.3. Proof of Proposition A.1}

Assume $(y, s)=(0,0)$ and for $n=0,1,2 \ldots$ set

$$
\rho_{n}=\sum_{j=1}^{n} \frac{1}{2^{j}} \rho, \quad K_{n}=K_{\rho_{n}} ; \quad \tilde{\rho}_{n}=\frac{\rho_{n}+\rho_{n+1}}{2}, \quad \tilde{K}_{n}=K_{\tilde{\rho}_{n}}
$$

and let $x \rightarrow \zeta_{n}(x)$ be a non-negative, piecewise smooth cutoff function in $\tilde{K}_{n}$ that equal one on $K_{n}$, and such that $\left|D \zeta_{n}\right| \leq 2^{n+2} / \rho$. In the weak formulation of (1.1)(1.3) take $\zeta_{n}$ as a test function, to obtain

$$
\begin{aligned}
\int_{\tilde{K}_{n}} u\left(x, \tau_{1}\right) \zeta_{n} d x \leq & \int_{\tilde{K}_{n}} u\left(x, \tau_{2}\right) \zeta_{n} d x \\
& +\left.\frac{2^{n+2}}{\rho}\left[C_{1}+(C \rho)\right]\left|\int_{\tau_{1}}^{\tau_{2}} \int_{\tilde{K}_{n}}\right| D u\right|^{p-1} d x d \tau \mid \\
& +2^{n+2+N}\left(\frac{C \rho}{\epsilon}\right)^{p-1}[1+(C \rho)]\left(\frac{t}{\rho^{\lambda}}\right)^{\frac{1}{2-p}}
\end{aligned}
$$

for any two time levels $\tau_{1}$ and $\tau_{2}$ in $[0, t]$, where $\epsilon$ is defined in (A.1). Therefore if $C$ violates the first of (A.1)

$$
\begin{aligned}
\int_{K_{n}} u\left(x, \tau_{1}\right) d x \leq & \int_{K_{2 \rho}} u\left(x, \tau_{2}\right) d x \\
& +\frac{\gamma 2^{n}}{\rho} \int_{0}^{t} \int_{\tilde{K}_{n}}|D u|^{p-1} d x d \tau+2^{n+2}\left(\frac{t}{\rho^{\lambda}}\right)^{\frac{1}{2-p}} .
\end{aligned}
$$

As time level $\tau_{2}$ take one for which

$$
\int_{K_{2 \rho}} u\left(x, \tau_{2}\right) d x=\inf _{0 \leq \tau \leq t} \int_{K_{2 \rho}} u(x, \tau) d x \stackrel{\text { def }}{=} \mathcal{I} .
$$


Also set

$$
\mathcal{S}_{n}=\sup _{0 \leq \tau \leq t} \int_{K_{n}} u(x, \tau) d x
$$

Since $\tau_{1} \in[0, t]$ is arbitrary, the previous inequality yields

$$
\mathcal{S}_{n} \leq \mathcal{I}+\frac{\gamma 2^{n}}{\rho} \int_{0}^{t} \int_{\tilde{K}_{n}}|D u|^{p-1} d x d \tau+\gamma 2^{n}\left(\frac{t}{\rho^{\lambda}}\right)^{\frac{1}{2-p}} .
$$

The term involving $|D u|$ is estimated above by applying (A.5) over the pair of cubes $\tilde{K}_{n} \subset K_{n+1}$ for which $(1-\sigma)=2^{-(n+2)}$, and for $\delta=\gamma^{-1} 2^{-n-2} \varepsilon_{o}$, where $\varepsilon_{o} \in(0,1)$ is to be chosen. For these choices

$$
\frac{2^{n+2}}{\rho} \int_{0}^{t} \int_{\tilde{K}_{n}}|D u|^{p-1} d x d \tau \leq \varepsilon_{o} \mathcal{S}_{n+1}+\gamma\left(p, \varepsilon_{o}\right) b^{n}\left(\frac{t}{\rho^{\lambda}}\right)^{\frac{1}{2-p}} \quad \text { where } b=2^{p^{2}}
$$

Combining these remarks gives the recursive inequalities

$$
\mathcal{S}_{n} \leq \varepsilon_{o} \mathcal{S}_{n+1}+\gamma\left(\text { data, } \varepsilon_{o}\right)\left\{\mathcal{I}+\left(\frac{t}{\rho^{\lambda}}\right)^{\frac{1}{2-p}}\right\}
$$

The theorem now follows from the interpolation Lemma 4.3 of [6, Chapter I].

\section{A.4. Proof of Theorem 2.1}

The theorem is an immediate consequence of Proposition A.1 and the following sup-estimate.

Proposition A.4. Let $u$ be a non-negative, weak solution to (1.1)-(1.3) for $p_{*}<$ $p<2$, or equivalently for $\lambda>0$. There exists a positive constant $\gamma$ depending only upon the data, such that for all cylinders $K_{\rho}(y) \times[2 s-t, t] \subset E_{T}$, either (A.1) holds, or

$$
\sup _{K_{\frac{1}{2} \rho}(y) \times[s, t]} u \leq \gamma\left(\frac{\rho^{p}}{t-s}\right)^{\frac{N}{\lambda}}\left(\frac{1}{\rho^{N}(t-s)} \int_{2 s-t}^{t} \int_{K_{\rho}(y)} u d x d \tau\right)^{\frac{p}{\lambda}}+\left(\frac{t-s}{\rho^{p}}\right)^{\frac{1}{2-p}} .
$$

Proof. Assume $(y, s)=(0,0)$ and for fixed $\sigma \in(0,1)$ and $n=0,1,2, \ldots$ set

$$
\begin{array}{ll}
\rho_{n}=\sigma \rho+\frac{1-\sigma}{2^{n}} \rho, & t_{n}=-\sigma t-\frac{1-\sigma}{2^{n}} t \\
K_{n}=K_{\rho_{n}}, & Q_{n}=K_{n} \times\left(t_{n}, t\right) .
\end{array}
$$


This is a family of nested and shrinking cylinders with common "vertex" at $(0, t)$, and by construction $Q_{o}=K_{\rho} \times(-t, t)$ and $Q_{\infty}=K_{\sigma \rho} \times(-\sigma t, t)$. By the results of [6, Chapter V], the solution $u$ is locally essentially bounded in $E_{T}$ and we set

$$
M=\underset{Q_{o}}{\operatorname{ess} \sup } u, \quad M_{\sigma}=\underset{Q_{\infty}}{\operatorname{ess} \sup } u .
$$

We first find a relationship between $M$ and $M_{\sigma}$. Denote by $\zeta$ a non-negative, piecewise smooth cutoff function in $Q_{n}$ that equals one on $Q_{n+1}$ and has the form $\zeta(x, t)=\zeta_{1}(x) \zeta_{2}(t)$, where

$$
\begin{aligned}
& \zeta_{1}=\left\{\begin{array}{ll}
1 & \text { in } K_{n+1} \\
0 & \text { in } \mathbb{R}^{N}-K_{n}
\end{array} \quad\left|D \zeta_{1}\right| \leq \frac{2^{n+1}}{(1-\sigma) \rho}\right. \\
& \zeta_{2}=\left\{\begin{array}{ll}
0 & \text { for } t \leq t_{n} \\
1 & \text { for } t \geq t_{n+1}
\end{array} \quad 0 \leq \zeta_{2, t} \leq \frac{2^{n+1}}{(1-\sigma) t}\right.
\end{aligned}
$$

introduce the increasing sequence of levels $k_{n}=k-2^{-n} k$, where $k>0$ is to be chosen, and in the weak formulation of (1.2), take the test functions $\left(u-k_{n+1}\right)_{+} \zeta^{p}$ to obtain, after standard calculations

$$
\begin{aligned}
& \sup _{t_{n} \leq \tau \leq t} \int_{K_{n}}\left[\left(u-k_{n+1}\right)_{+} \zeta\right]^{2}(x, \tau) d x+\iint_{Q_{n}}\left|D\left[\left(u-k_{n+1}\right)_{+} \zeta\right]\right|^{p} d x d \tau \\
& \leq \frac{\gamma 2^{n p}}{(1-\sigma)^{p} \rho^{p}}\left[1+(C \rho)^{p}\right] \iint_{Q_{n}}\left(u-k_{n}\right)_{+}^{p} d x d \tau \\
& \quad+\frac{\gamma 2^{n}}{(1-\sigma) t} \iint_{C_{n}}\left(u-k_{n}\right)_{+}^{2} d x d \tau+\gamma C^{p} \iint_{Q_{n}} \chi_{\left[\left(u-k_{n}\right)_{+}>0\right]} d x d \tau .
\end{aligned}
$$

Estimate

$$
\begin{gathered}
\iint_{Q_{n}}\left(u-k_{n}\right)_{+}^{p} d x d \tau \leq M^{p-1} \iint_{Q_{n}}\left(u-k_{n}\right)_{+} d x d \tau \\
\iint_{Q_{n}}\left(u-k_{n}\right)_{+}^{2} d x d \tau \leq M \iint_{Q_{n}}\left(u-k_{n}\right)_{+} d x d \tau \\
\iint_{Q_{n}} \chi_{\left[\left(u-k_{n+1}\right)_{+}>0\right]} d x d \tau \leq \frac{2^{n+1}}{k} \iint_{Q_{n}}\left(u-k_{n}\right)_{+} d x d \tau .
\end{gathered}
$$

Therefore stipulating that $C \rho$ violates (A.1), the previous inequalities yield

$$
\begin{aligned}
& \sup _{t_{n} \leq \tau \leq t} \int_{K_{n}}\left[\left(u-k_{n+1}\right)_{+} \zeta\right]^{2}(x, \tau) d x+\iint_{Q_{n}}\left|D\left[\left(u-k_{n+1}\right)_{+} \zeta\right]\right|^{p} d x d \tau \\
& \leq \frac{\gamma 2^{n p} M}{(1-\sigma)^{p} t}\left\{1+\left(\frac{t}{\rho^{p}}\right) M^{p-2}+\frac{(C \rho)^{p}}{M k}\left(\frac{t}{\rho^{p}}\right)\right\} \iint_{Q_{n}}\left(u-k_{n}\right)_{+} d x d \tau .
\end{aligned}
$$


The last term in $\{\cdots\}$ is estimated by stipulating to take

$$
k \leq M \quad \text { and } \quad k \geq\left(\frac{t}{\rho^{p}}\right)^{\frac{1}{2-p}} .
$$

With these stipulations, taking into account the restriction (A.1) on $C \rho$, and that $1<p<2$, estimate

$$
\{\cdots\} \leq 1+\frac{1}{k^{2-p}}\left(\frac{t}{\rho^{p}}\right)+\frac{1}{k^{2}}\left(\frac{t}{\rho^{p}}\right)^{\frac{p}{2-p}}\left(\frac{t}{\rho^{p}}\right) \leq 3 .
$$

Therefore, if (A.1) is violated and (A.6) is in force, there exists a constant $\gamma=$ $\gamma$ (data) such that

$$
\begin{aligned}
\sup _{t_{n} \leq \tau \leq t} \int_{K_{n}}\left[\left(u-k_{n+1}\right)_{+} \zeta\right]^{2}(x, \tau) d x & +\iint_{Q_{n}}\left|D\left[\left(u-k_{n+1}\right)_{+} \zeta\right]\right|^{p} d x d \tau \\
& \leq \frac{\gamma 2^{n p} M}{(1-\sigma)^{p} t} \iint_{Q_{n}}\left(u-k_{n}\right)_{+} d x d \tau .
\end{aligned}
$$

By Hölder's inequality and the embedding Proposition 3.1 of [6, Chapter I]

$$
\begin{aligned}
& \iint_{Q_{n+1}}\left(u-k_{n+1}\right)_{+} d x d \tau \leq\left(\iint_{Q_{n}}\left[\left(u-k_{n+1}\right)_{+} \zeta\right]^{p \frac{N+2}{N}} d x d \tau\right)^{\frac{N}{p(N+2)}} \\
& \quad \times\left(\iint_{Q_{n}} \chi_{\left[\left(u-k_{n+1}\right)_{+}>0\right]} d x d \tau\right)^{1-\frac{N}{p(N+2)}} \\
& \leq \gamma\left(\iint_{Q_{n}}\left|D\left[\left(u-k_{n+1}\right)_{+} \zeta\right]\right|^{p} d x d \tau\right)^{\frac{N}{p(N+2)}} \\
& \quad \times\left(\sup _{t_{n} \leq \tau \leq t} \int_{K_{n}}\left[\left(u-k_{n}\right)_{+} \zeta\right]^{2}(x, \tau) d x\right)^{\frac{1}{N+2}} \\
& \quad \times\left(\iint_{Q_{n}} \chi_{\left[\left(u-k_{n+1}\right)_{+}>0\right]} d x d \tau\right)^{1-\frac{N}{p(N+2)}} \\
& \leq \gamma\left(\frac{2^{p n} M}{(1-\sigma)^{p} t}\right)^{\frac{1}{p} \frac{N+p}{N+2}}\left(\iint_{Q_{n}}\left(u-k_{n}\right)_{+} d x d \tau\right)^{\frac{N}{p(N+2)}+\frac{1}{N+2}} \\
& \quad \times\left(\iint_{Q_{n}} \chi_{\left[\left(u-k_{n+1}\right)_{+}>0\right]} d x d \tau\right)^{1-\frac{N}{p(N+2)}} .
\end{aligned}
$$

Estimate

$$
\iint_{Q_{n}} \chi_{\left[\left(u-k_{n+1}\right)_{+}>0\right]} d x d \tau \leq \frac{2^{n+1}}{k} \iint_{Q_{n}}\left(u-k_{n}\right)_{+} d x d \tau
$$


and set

$$
Y_{n}=\frac{1}{\left|Q_{n}\right|} \iint_{Q_{n}}\left(u-k_{n}\right)_{+} d x d \tau=\iint_{Q_{n}}\left(u-k_{n}\right)_{+} d x d \tau .
$$

Then the previous recursive inequalities can be written more concisely as

$$
Y_{n+1} \leq \frac{\gamma b^{n}}{(1-\sigma)^{\frac{N+p}{N+2}}}\left(\frac{M^{N+p}}{k^{N+p+\lambda}}\right)^{\frac{1}{p(N+2)}}\left(\frac{\rho^{p}}{t}\right)^{\frac{N}{p} \frac{1}{N+2}} Y_{n}^{1+\frac{1}{N+2}}
$$

for $b=b(N, p)>1$. It follows from these that $\left\{Y_{n}\right\} \rightarrow 0$ as $n \rightarrow \infty$ provided $k$ is chosen to satisfy

$$
Y_{o}=\iint_{Q_{o}} u d x d \tau=\frac{(1-\sigma)^{N+p}}{\gamma^{N+2} b^{(N+2)^{2}}}\left(\frac{k^{N+p+\lambda}}{M^{N+p}}\right)^{\frac{1}{p}}\left(\frac{t}{\rho^{p}}\right)^{\frac{N}{p}} .
$$

For these choices

$$
M_{\sigma} \leq \frac{\gamma(\text { data })}{(1-\sigma)^{\frac{p(N+p)}{N+p+\lambda}}} M^{\frac{N+p}{N+p+\lambda}}\left(\frac{\rho^{p}}{t}\right)^{\frac{N}{N+p+\lambda}}\left(\int_{Q_{o}} u d x d \tau\right)^{\frac{p}{N+p+\lambda}}
$$

From this, by a standard interpolation process and taking into account (A.6)

$$
\sup _{K_{\frac{1}{2} \rho} \times[0, t]} u \leq \gamma(\text { data })\left(\frac{\rho^{p}}{t}\right)^{\frac{N}{\lambda}}\left(\int_{Q_{o}} u d x d \tau\right)^{\frac{p}{\lambda}}+\left(\frac{t}{\rho^{p}}\right)^{\frac{1}{2-p}} .
$$

\section{B. Proof of Theorem 2.3}

\section{B.1. Energy estimates for the truncations $(u-k)_{ \pm}$}

For $\rho>0$ and $\theta>0$ set

$$
Q_{\rho}^{-}(\theta)=K_{\rho} \times\left(-(\theta \rho)^{p}, 0\right], \quad Q_{\rho}^{+}(\theta)=K_{\rho} \times\left(0,(\theta \rho)^{p}\right]
$$

and for $(y, s) \in \mathbb{R}^{N} \times \mathbb{R}$

$$
\begin{aligned}
& (y, s)+Q_{\rho}^{-}(\theta)=K_{\rho}(y) \times\left(s-(\theta \rho)^{p}, s\right] \\
& (y, s)+Q_{\rho}^{+}(\theta)=K_{\rho}(y) \times\left(s, s+(\theta \rho)^{p}\right] .
\end{aligned}
$$

We also write $Q_{\rho}^{ \pm}(1)=Q_{\rho}^{ \pm}$. There exists a constant $\gamma=\gamma$ (data) such that, for every cylinder $\left[(y, s)+Q_{\rho}^{-}(\theta)\right] \subset E_{T}$, every $k \in \mathbb{R}$ and every piecewise smooth, 
non-negative function $\zeta$ vanishing on $\partial K_{\rho}(y)$

$$
\begin{aligned}
& \underset{s-(\theta \rho)^{p}<t<s}{\operatorname{ess} \sup _{S(y)}} \int_{K_{\rho}(u-k)_{ \pm}^{2} \zeta^{p}(x, t) d x}(u \\
& -\int_{K_{\rho}(y)}(u-k)_{ \pm}^{2} \zeta^{p}\left(x, s-(\theta \rho)^{p}\right) d x \\
& +C_{o} \iint_{(y, s)+Q_{\rho}^{-}(\theta)}\left|D(u-k)_{ \pm} \zeta\right|^{p} d x d \tau \\
& \leq \gamma \iint_{(y, s)+Q_{\rho}^{-}(\theta)}\left[(u-k)_{ \pm}^{p}\left(|D \zeta|^{p}+C^{p}\right)+(u-k)_{ \pm}^{2}\left|\zeta_{\tau}\right|\right] d x d \tau \\
& +\gamma C^{p} \iint_{(y, s)+Q_{\rho}^{-}(\theta)} \chi_{\left[(u-k)_{ \pm}>0\right]} \zeta^{p} d x d \tau
\end{aligned}
$$

where $C_{o}$ and $C$ are the constants appearing in the structure conditions (1.3). These energy estimates are proved by taking $\pm(u-k)_{ \pm} \zeta^{p}$ in the weak formulation of (1.1) and carrying standard calculations. Similar energy estimates hold for cylinders $\left[(y, s)+Q_{\rho}^{+}(\theta)\right] \subset E_{T}$.

\section{B.2. A De Giorgi-type lemma}

For a fixed cylinder $\left[(y, s)+Q_{2 \rho}^{-}(\theta)\right] \subset E_{T}$, denote by $\mu_{ \pm}$and $\omega$, non-negative numbers such that

$$
\mu_{+} \geq \operatorname{ess} \sup _{(y, s)+Q_{2 \rho}^{-}(\theta)} u, \quad \mu_{-} \leq \operatorname{essinf}_{(y, s)+Q_{2 \rho}^{-}(\theta)}^{\operatorname{en}} u, \quad \omega \geq \mu_{+}-\mu_{-} .
$$

Denote by $\xi$ and $a$ fixed numbers in $(0,1)$.

Lemma B.1. There exists a number $v$ depending upon the data and $\theta, \xi$, $\omega$ and $a$, such that if

$$
\left|\left[u \geq \mu_{+}-\xi \omega\right] \cap\left[(y, s)+Q_{2 \rho}^{-}(\theta)\right]\right| \leq v\left|Q_{2 \rho}^{-}(\theta)\right|
$$

then either

$$
C \rho>\min \{1 ; \xi \omega\}
$$

or

$$
u \leq \mu_{+}-a \xi \omega \quad \text { a.e. in }\left[(y, s)+Q_{\rho}^{-}(\theta)\right]
$$

Likewise, if

$$
\left|\left[u \leq \mu_{-}+\xi \omega\right] \cap\left[(y, s)+Q_{2 \rho}^{-}(\theta)\right]\right| \leq \nu\left|Q_{2 \rho}^{-}(\theta)\right|
$$

then either (B.3) holds true, or

$$
u \geq \mu_{-}+a \xi \omega \quad \text { a.e. in }\left[(y, s)+Q_{\rho}^{-}(\theta)\right] .
$$


Proof. We prove the second statement, to trace the precise dependence of $v$ on $\theta$, $a, \xi$ and $\omega$. Assume $(y, s)=(0,0)$, and for $n=0,1,2, \ldots$, set

$$
\rho_{n}=\rho+\frac{\rho}{2^{n}}, \quad K_{n}=K_{\rho_{n}}, \quad Q_{n}=K_{n} \times\left(-\left(\theta \rho_{n}\right)^{p}, 0\right] .
$$

Apply (B.1) over $K_{n}$ and $Q_{n}$ to $\left(u-k_{n}\right)_{-}$, for the levels

$$
k_{n}=\mu_{-}+\xi_{n} \omega \quad \text { where } \quad \xi_{n}=a \xi+\frac{1-a}{2^{n}} \xi .
$$

The cutoff function $\zeta$ is taken of the form $\zeta(x, t)=\zeta_{1}(x) \zeta_{2}(t)$, where

$$
\begin{aligned}
& \zeta_{1}=\left\{\begin{array}{ll}
1 & \text { in } K_{n+1} \\
0 & \text { in } \mathbb{R}^{N}-K_{n}
\end{array} \quad\left|D \zeta_{1}\right| \leq \frac{1}{\rho_{n}-\rho_{n+1}}=\frac{2^{n+1}}{\rho}\right. \\
& \zeta_{2}=\left\{\begin{array}{ll}
0 & \text { for } t<-\theta^{p} \rho_{n}^{p} \\
1 & \text { for } t \geq-\theta^{p} \rho_{n+1}^{p}
\end{array} \quad 0 \leq \zeta_{2, t} \leq \frac{1}{\theta^{p}\left(\rho_{n}^{p}-\rho_{n+1}^{p}\right)} \leq \frac{2^{p(n+1)}}{(\theta \rho)^{p}} .\right.
\end{aligned}
$$

With these stipulations, and assuming that (B.3) is violated, the energy inequalities (B.1) yield

$$
\begin{aligned}
& \operatorname{ess~sup}_{-\left(\theta \rho_{n}\right)^{p}<t<0} \int_{K_{n}}\left(u-k_{n}\right)_{-}^{2} \zeta^{2}(x, t) d x+\iint_{Q_{n}}\left|D\left(u-k_{n}\right)_{-} \zeta\right|^{p} d x d \tau \\
& \leq \gamma \frac{2^{n p}}{\rho^{p}}\left(\iint_{Q_{n}}\left(u-k_{n}\right)_{-}^{p} d x d \tau+\frac{1}{\theta^{p}} \iint_{Q_{n}}\left(u-k_{n}\right)_{-}^{2} d x d \tau\right) \\
& \leq \gamma \frac{2^{n p}}{\rho^{p}}\left((\xi \omega)^{p}+\frac{(\xi \omega)^{2}}{\theta^{p}}\right)\left|\left[u<k_{n}\right] \cap Q_{n}\right|
\end{aligned}
$$

By Proposition 3.1 of [6, Chapter I],

$$
\begin{aligned}
& \iint_{Q_{n}}\left[\left(u-k_{n}\right)_{-} \zeta\right]^{p \frac{N+2}{N}} d x d t \\
& \leq \iint_{Q_{n+1}}\left|D\left[\left(u-k_{n}\right)_{-} \zeta\right]\right|^{p} d x d \tau\left(\operatorname{ess}_{-\left(\theta \rho_{n}\right)^{p}<t<0} \int_{K_{n}(t)}\left[\left(u-k_{n}\right)_{-} \zeta\right]^{2} d x\right)^{\frac{p}{N}} \\
& \leq \gamma\left[\frac{2^{n p}}{\rho^{p}}\left((\xi \omega)^{p}+\frac{(\xi \omega)^{2}}{\theta^{p}}\right)\right]^{\frac{N+p}{N}}\left|\left[u<k_{n}\right] \cap Q_{n}\right|^{\frac{N+p}{N}} .
\end{aligned}
$$

Estimate below

$$
\iint_{Q_{n}}\left[\left(u-k_{n}\right)_{-} \zeta\right]^{p \frac{N+2}{N}} d x d t \geq\left[2^{-(n+1)}(1-a) \xi \omega\right]^{p \frac{N+2}{N}}\left|\left[u<k_{n+1}\right] \cap Q_{n+1}\right|
$$


and set

$$
Y_{n}=\frac{\left|\left[u<k_{n}\right] \cap Q_{n}\right|}{\left|Q_{n}\right|}
$$

Then

$$
Y_{n+1} \leq \frac{\gamma b^{n}}{(1-a)^{(N+2) \frac{p}{N}}}\left(\frac{\theta^{p}}{(\xi \omega)^{2-p}}\right)^{\frac{p}{N}}\left[1+\frac{(\xi \omega)^{2-p}}{\theta^{p}}\right]^{\frac{N+p}{N}} Y_{n}^{1+\frac{p}{N}}
$$

where

$$
b=2^{\frac{p}{N}[2(N+1)+p]} .
$$

By Lemma 4.1 of [6, Chapter I], $\left\{Y_{n}\right\} \rightarrow 0$ as $n \rightarrow \infty$, provided

$$
Y_{o} \leq \gamma_{o}(1-a)^{N+2} \frac{\frac{(\xi \omega)^{2-p}}{\theta^{p}}}{\left(1+\frac{(\xi \omega)^{2-p}}{\theta^{p}}\right)^{\frac{N+p}{p}}} \stackrel{\text { def }}{=} v
$$

Thus this choice of $v$ yields $Y_{\infty}=0$ which is equivalent to (B.6). Similar arguments for the corresponding statement (B.2)-(B.4) yield the same expression in (B.7) with the proper interpretation of $Y_{o}$.

\section{B.3. Proof of Theorem 2.3. Transforming $u$ into $w$}

Regard the equation as holding in $K_{8 \rho}(y) \times\left(s, s+(\delta \theta \rho)^{p}\right]$ and introduce the change of variables and the new unknown

$$
z=\frac{x-y}{\rho}, \quad \tau=\frac{t-s}{\delta \theta^{p} \rho^{p}}, \quad w(z, \tau)=\frac{2}{M} u(x, t) .
$$

The cylindrical domain $K_{8 \rho}(y) \times\left(s, s+(\delta \theta \rho)^{p}\right]$ is transformed into $K_{8} \times(0,1]$, and by formal calculations

$$
w_{\tau}-\operatorname{div}_{z} \tilde{\mathbf{A}}\left(z, \tau, w, D_{z} w\right)=\tilde{B}\left(z, \tau, w, D_{z} w\right)
$$

in the weak sense over $K_{8} \times(0,1]$. Assuming that $C \rho \leq 2 \min \{1 ; M\}$, the functions $\tilde{\mathbf{A}}$ and $\tilde{B}$ satisfy the structure conditions

$$
\left\{\begin{array}{l}
\tilde{\mathbf{A}}(x, \tau, w, D w) \cdot D w \geq 2^{-p} \delta C_{o}|D w|^{p}-\delta\left(\frac{C \rho}{2 M}\right)^{p} \\
|\tilde{\mathbf{A}}(x, \tau, w, D w)| \leq 2^{-p} \delta C_{1}|D w|^{p-1}+\delta\left(\frac{C \rho}{2 M}\right)^{p-1} \\
|\tilde{B}(x, \tau, w, D w)| \leq 2^{-p} \delta C \rho|D w|^{p-1}+\delta(C \rho)\left(\frac{C \rho}{2 M}\right)^{p-1}
\end{array}\right.
$$


where $C_{o}$ and $C_{1}$ are the constants appearing in (1.3). These coefficients depend upon $\delta$, which is assumed to be a parameter quantitatively determined only in terms of the data. Assume momentarily

$$
w_{\tau} \in C\left[(0,1] ; L^{1}\left(K_{8}\right)\right] .
$$

Then (B.8)-(B.9) and the structure assumption (1.2)_ imply the weak inequality

$$
\begin{aligned}
\int_{K_{8}} \frac{\partial}{\partial \tau}(k-w)_{+} \varphi d z & +\int_{K_{8}} \tilde{A}\left(z, \tau,(k-w)_{+}, D_{z}(k-w)_{+}\right) \cdot D \varphi d z \\
& \leq \int_{K_{8}} \tilde{B}\left(z, \tau,(k-w)_{+}, D_{z}(k-w)_{+}\right) \varphi d z
\end{aligned}
$$

for all admissible non-negative test functions $\varphi$. The assumption (2.5) of Theorem 2.3, translates into

$$
\left|[w(\cdot, \tau) \geq 1] \cap K_{1}\right|>\alpha \quad \text { for all } \tau \in(0,1] .
$$

This measure-theoretical lower bound will be converted into a lower bound for $w$ over the expanded cube $K_{2}$ for all "times" $\tau \in\left(\frac{1}{2}, 1\right]$.

Lemma B.2. Let $w$ be a weak solution of (B.8)-(B.9). For every $v \in(0,1)$ there exists $\epsilon_{v}$ depending only upon the data, $v$ and $\alpha$, such that either

$$
\delta^{\frac{1}{p}} C \rho>\min \left\{1 ; 2 \epsilon_{v} M\right\}
$$

or

$$
\left|\left[w(\cdot, \tau)<\epsilon_{\nu}\right]\right| \leq \nu\left|K_{4}\right| \quad \text { for all } \tau \in\left(\frac{1}{4}, 1\right] .
$$

B.3.1. Proof of Theorem 2.3 assuming Lemma B.2

Apply (B.5)-(B.6) of Lemma B.1 to $w$, with $\mu_{-}=0, \xi=1$ and $\omega=\epsilon_{v}$ over the cylinders

$$
K_{4} \times\left(\tau, \tau+\tilde{\theta}^{p}\right] \quad \text { where } \tilde{\theta}^{p}=\epsilon_{v}^{2-p} .
$$

These are contained in $K_{4} \times\left(\frac{1}{4}, 1\right]$ as $\tau$ ranges over $\left(\frac{1}{4}, 1-\tilde{\theta}^{p}\right]$. In (B.7) choose $a=\frac{1}{2}$. This determines, quantitatively, only in terms of the data, the number $v \in(0,1)$ for which

$$
w(\cdot, \tau) \geq \frac{1}{2} \epsilon_{v} \quad \text { in } K_{2} \quad \text { for all } \tau \in\left(\frac{1}{2}, 1\right] .
$$

The expansion of positivity of Theorem 2.3 follows from this, upon returning to the original coordinates. 


\section{B.4. Proof of Lemma B.2}

Let $\zeta$ be a cutoff function in $K_{8}$ that equals one on $K_{4}$, vanishes outside $K_{8}$, such that $|D \zeta| \leq 4$ and the sets $[\zeta \geq k]$ are convex for all $k \in(0,1)$. In the weak inequality (B.11) take the testing function

$$
\varphi=\frac{\zeta^{p} \tau}{\left[k-(k-w)_{+}+\epsilon k\right]^{p-1}}
$$

where $k, \epsilon \in(0,1)$ are to be chosen. In the arguments to follow, the product $k \epsilon$ will form the number $\epsilon_{v}$ claimed by Lemma B.2. Accordingly it is stipulated that the various choices of $k \epsilon$ below will violate (B.13). Thus for $q>0$

$$
\left(\frac{C \rho}{2 M}\right)^{q} \frac{1}{\left[k-(k-w)_{+}+\epsilon k\right]^{q}} \leq\left(\frac{C \rho}{2 \epsilon k M}\right)^{q} \leq \delta^{-\frac{q}{p}} .
$$

Set also

$$
\begin{aligned}
& \Phi_{k}(w)=\int_{0}^{(k-w)_{+}} \frac{d s}{[k-s+\epsilon k]^{p-1}} \\
& \Psi_{k}(w)=\ln \frac{k(1+\epsilon)}{k(1+\epsilon)-(k-w)_{+}} .
\end{aligned}
$$

By standard calculations, there exist constants $\bar{\gamma}_{o}$ and $\bar{\gamma}$, depending only upon the data, such that

$$
\frac{d}{d \tau} \int_{K_{8}} \Phi_{k}(w) \zeta^{p} \tau d z+\bar{\gamma}_{o} \int_{K_{8}}\left|D \Psi_{k}(w)\right|^{p} \zeta^{p} \tau d z \leq \frac{\bar{\gamma}}{2-p} .
$$

By virtue of (B.12) and the embedding Proposition 2.1 of Chapter I of [6]

$$
\int_{K_{8}} \Psi_{k}^{p}\left[(w(z, \tau)] \zeta^{p} d z \leq C_{N} \int_{K_{8}}\left|D \Psi_{k}[w(z, \tau)]\right|^{p} \zeta^{p} d z\right.
$$

for a constant $C_{N}$ depending only upon the data, and for all $\tau \in(0,1]$. Therefore there exist constants $\gamma_{o}$ and $\gamma$, depending only upon the data, such that

$$
\frac{d}{d \tau} \int_{K_{8}} \Phi_{k}[w(z, \tau)] \zeta^{p} \tau d z+\gamma_{o} \int_{K_{8}} \Psi_{k}[w(z, \tau)]^{p} \zeta^{p} \tau d z \leq \gamma .
$$

Introduce the quantities

$$
Y_{n}=\sup _{0<\tau \leq 1} \int_{K_{8}} \zeta^{p}(x) \tau \chi\left[w(\cdot, \tau)<\epsilon^{n}\right] d z \quad \text { for } n=0,1, \ldots
$$

The proof of Lemma B.2 is a consequence of the following

Proposition B.3. For every $v \in(0,1)$, there exist numbers $\epsilon$ and $\sigma$ in $(0,1)$ depending only upon $N$, $p$, the data and $v$, such that if (B.13) is violated for $\epsilon_{v}=\epsilon^{n}$, then either

$$
Y_{n} \leq \frac{1}{2} v \quad \text { or } \quad Y_{n+1} \leq \max \left\{\frac{1}{2} \nu ; \sigma Y_{n}\right\} .
$$


B.4.1. Proof of Lemma B.2, Assuming Proposition B.3

Iterating (B.18) gives

$$
Y_{n} \leq \max \left\{\frac{1}{2} \nu ; \sigma^{n-1} Y_{o}\right\} \quad n=1,2, \ldots
$$

Since $Y_{o} \leq\left|K_{8}\right|$, take $n=n_{o}$ so large that $2 \sigma^{n_{o}-1} \leq \nu 8^{-N}$. Then the lemma follows with $\epsilon_{v}=\sigma^{n_{o}}$.

\section{B.5. Proof of Proposition B.3}

In (B.16) take $k=\epsilon^{n}$ for $n \in \mathbb{N}$, where $\epsilon \in(0,1)$ is to be chosen. By the definition of $Y_{n}$, for every $\varepsilon$ there exists $\tau_{o} \in(0,1)$, such that

$$
\int_{K_{8}} \zeta^{p}(x) \tau_{o} \chi_{\left[w\left(\cdot, \tau_{o}\right)<\epsilon^{n+1}\right]} d z \geq Y_{n+1}-\varepsilon .
$$

The numbers $n$ and $\tau_{o} \in(0,1)$ being fixed, consider the following two cases. Either

$$
\frac{d}{d t} \int_{K_{8}} \zeta^{p} \tau_{o} \Phi_{\epsilon^{n}}\left[w\left(z, \tau_{o}\right)\right] d z \geq 0
$$

or

$$
\frac{d}{d t} \int_{K_{8}} \zeta^{p} \tau_{o} \Phi_{\epsilon^{n}}\left[w\left(z, \tau_{o}\right)\right] d z<0
$$

In either case assume that $Y_{n}>\frac{1}{2} \nu$, otherwise the conclusion is trivial. While $\varepsilon$ in (B.19) is arbitrary, it will be taken in the range $\varepsilon \in\left(0, \frac{1}{4} v\right)$.

If (B.20) holds, then from (B.16) with $k=\epsilon^{n}$

$$
\int_{K_{8}} \zeta^{p} \tau_{o} \Psi_{\epsilon^{n}}\left[w\left(z, \tau_{o}\right)\right] d z \leq \frac{\gamma}{\gamma_{o}}
$$

Minorize this integral by extending the integration over the smaller set

$$
\left[w\left(\cdot, t_{o}\right)<\epsilon^{n+1}\right] \cap K_{8} \quad \text { on which } \quad \Psi_{\epsilon^{n}}(w) \geq \ln \frac{\epsilon^{n}(1+\epsilon)}{2 \epsilon^{n+1}} .
$$

Therefore

$$
\left(\ln \frac{1+\epsilon}{2 \epsilon}\right)^{p} \int_{K_{8} \cap\left[w\left(\cdot, \tau_{o}\right)<\epsilon^{n+1}\right]} \zeta^{p} \tau_{o} d z \leq \int_{K_{8}} \Psi_{\epsilon^{n}}\left[w\left(z, \tau_{o}\right)\right] \zeta^{p} \tau_{o} d z
$$


From this and (B.19)

$$
Y_{n+1} \leq \varepsilon+\frac{\gamma}{\gamma_{o}}\left(\ln \frac{1+\epsilon}{2 \epsilon}\right)^{-p} \leq \frac{1}{2} v
$$

for the choices $\varepsilon \leq \frac{1}{4} \epsilon$ and then for $\epsilon$ so small that

$$
\frac{\gamma}{\gamma_{o}}\left(\ln \frac{1+\epsilon}{2 \epsilon}\right)^{-p} \leq \frac{1}{4} \nu
$$

Such a choice depends only upon the constants $\gamma, \gamma_{o}$ and $v$ and therefore it depends only upon the data.

\section{B.6. Proof of Proposition B.3 when (B.10) holds}

If (B.10) holds true, define

$$
\tau_{*}=\sup \left\{\tau \in\left(0, \tau_{o}\right) \mid \frac{d}{d t} \int_{K_{8}} \zeta^{p} \tau \Phi_{\epsilon^{n}}[w(z, \tau)] d z \geq 0\right\} .
$$

By the definition of $\tau_{*}$

$$
\int_{K_{8}} \zeta^{p} \tau_{o} \Phi_{\epsilon^{n}}\left[w\left(z, \tau_{o}\right)\right] d z \leq \int_{K_{8}} \zeta^{p} \tau_{*} \Phi_{\epsilon^{n}}\left[w\left(z, \tau_{*}\right)\right] d z .
$$

By the arguments of the first alternative

$$
\int_{K_{8}} \zeta^{p} \tau_{*} \Psi_{\epsilon^{n}}\left[w\left(z, \tau_{*}\right)\right] d z \leq \frac{\gamma}{\gamma_{o}}
$$

and for all $s \in(0,1)$

$$
\int_{K_{8}} \zeta^{p} \tau_{*} \chi_{\left[\left(\epsilon^{n}-w\right)_{+}>s \epsilon^{n}\right]} d z \leq \frac{\gamma}{\gamma_{o}}\left(\ln \frac{1+\epsilon}{1+\epsilon-s}\right)^{-p}
$$

From this and the definition of $Y_{n}$

$$
\begin{aligned}
\int_{K_{8}} \zeta^{p} \tau_{*} \chi_{\left[\left(\epsilon^{n}-w\right)_{+}>s \epsilon^{n}\right]} d z & \leq \min \left\{Y_{n} ; \frac{\gamma}{\gamma_{o}}\left(\ln \frac{1+\epsilon}{1+\epsilon-s}\right)^{-p}\right\} \\
& = \begin{cases}Y_{n} \\
\frac{\gamma}{\gamma_{o}}\left(\ln \frac{1+\epsilon}{1+\epsilon-s}\right)^{-p} \text { if } 0 \leq s<s_{*}\end{cases}
\end{aligned}
$$


where $s_{*}$ is the root of the equation

$$
Y_{n}=\frac{\gamma}{\gamma_{o}}\left(\ln \frac{1+\epsilon}{1+\epsilon-s}\right)^{-p} \Longrightarrow s_{*}=\frac{e^{\left(\frac{\gamma}{\gamma_{o}} \frac{1}{Y_{n}}\right)^{\frac{1}{p}}}-1}{e^{\left(\frac{\gamma}{\gamma_{o}} \frac{1}{Y_{n}}\right)^{\frac{1}{p}}}(1+\epsilon) .}
$$

Since $Y_{n}>v$, estimate

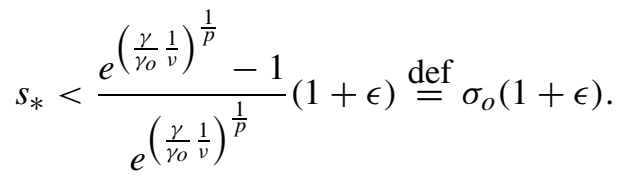

Next estimate the integral on the right hand side of (B.22). By Fubini's theorem and (B.23)-(B.24)

$$
\begin{aligned}
& \int_{K_{8}} \zeta^{p} \tau_{*} \Phi_{\epsilon^{n}}\left[w\left(z, \tau_{*}\right)\right] d z=\int_{K_{8}} \zeta^{p} \tau_{*}\left(\int_{0}^{\epsilon^{n}} \frac{\chi\left[s<\left(\epsilon^{n}-w\right)_{+}\right]}{\left[\epsilon^{n}(1+\epsilon)-s\right]^{p-1}} d s\right) d z \\
& =\int_{0}^{\epsilon^{n}} \frac{1}{\left[\epsilon^{n}(1+\epsilon)-s\right]^{p-1}} \int_{K_{8}} \zeta^{p} \tau_{*} \chi_{\left[\left(\epsilon^{n}-w\right)_{+}>s \epsilon^{n}\right]} d z d s \\
& =\int_{0}^{1} \frac{\epsilon^{n(2-p)}}{[1+\epsilon-s]^{p-1}} \int_{K_{8}} \zeta^{p} \tau_{*} \chi_{\left[\left(\epsilon^{n}-w\right)_{+}>s \epsilon^{n}\right]} d z d s \\
& =\int_{0}^{s_{*}} \frac{\epsilon^{n(2-p)}}{[1+\epsilon-s]^{p-1}} \int_{K_{8}} \zeta^{p} \tau_{*} \chi_{\left[\left(\epsilon^{n}-w\right)_{+}>s \epsilon^{n}\right]} d z d s \\
& +\int_{s_{*}}^{1} \frac{\epsilon^{n(2-p)}}{[1+\epsilon-s]^{p-1}} \int_{K_{8}} \zeta^{p} \tau_{*} \chi\left[\left(\epsilon^{n}-w\right)_{+}>s \epsilon^{n}\right] d z d s \\
& \leq \int_{0}^{s_{*}} \frac{\epsilon^{n(2-p)}}{[1+\epsilon-s]^{p-1}} Y_{n} d s \\
& +\int_{s_{*}}^{1} \frac{\epsilon^{n(2-p)}}{[1+\epsilon-s]^{p-1}} \frac{\gamma}{\gamma_{o}}\left(\ln \frac{1+\epsilon}{1+\epsilon-s}\right)^{-p} d s \\
& =\int_{0}^{1} \frac{\epsilon^{n(2-p)}}{[1+\epsilon-s]^{p-1}} Y_{n} d s \\
& -\int_{S_{*}}^{1}\left\{Y_{n}-\frac{\gamma}{\gamma_{o}}\left(\ln \frac{1+\epsilon}{1+\epsilon-s}\right)^{-p}\right\} \frac{\epsilon^{n(2-p)}}{[1+\epsilon-s]^{p-1}} d s \\
& =Y_{n} \epsilon^{n(2-p)} F\left(Y_{n}, \epsilon\right)
\end{aligned}
$$


where

$$
\begin{aligned}
F\left(Y_{n}, \epsilon\right)= & \int_{0}^{1} \frac{d s}{[1+\epsilon-s]^{p-1}} \\
& -\int_{s_{*}}^{1}\left[1-\frac{1}{Y_{n}} \frac{\gamma}{\gamma_{o}}\left(\ln \frac{1+\epsilon}{1+\epsilon-s}\right)^{-p}\right] \frac{d s}{[1+\epsilon-s]^{p-1}} \\
\leq & \int_{0}^{1} \frac{d s}{[1+\epsilon-s]^{p-1}} \\
& -\int_{\sigma_{o}(1+\epsilon)}^{1}\left[1-\frac{1}{v} \frac{\gamma}{\gamma_{o}}\left(\ln \frac{1+\epsilon}{1+\epsilon-s}\right)^{-p}\right] \frac{d s}{[1+\epsilon-s]^{p-1}}
\end{aligned}
$$

since $Y_{n} \geq v$ and by the definition (B.23)-(B.24) of $\sigma_{o}$. Combining these estimates, gives an upper bound for the right hand side of (B.22). Therefore

$$
\int_{K_{8}} \zeta^{p} \tau_{o} \Phi_{\epsilon^{n}}\left[w\left(z, \tau_{o}\right)\right] d z \leq Y_{n}[1-f(\epsilon)] \int_{0}^{1-\epsilon} \frac{\epsilon^{n(2-p)}}{[1+\epsilon-s]^{p-1}} d s
$$

where

$$
\begin{aligned}
& f(\epsilon) \int_{0}^{1-\epsilon} \frac{d s}{[1+\epsilon-s]^{p-1}}=-\int_{1-\epsilon}^{1} \frac{d s}{[1+\epsilon-s]^{p-1}} \\
& +\int_{\sigma_{o}(1+\epsilon)}^{1}\left[1-\frac{1}{v} \frac{\gamma}{\gamma_{o}}\left(\ln \frac{1+\epsilon}{1+\epsilon-s}\right)^{-p}\right] \frac{d s}{[1+\epsilon-s]^{p-1}} .
\end{aligned}
$$

Estimating below the left hand side of (B.25), gives

$$
\begin{aligned}
\int_{K_{8}} \zeta^{p} \tau_{o} \Phi_{\epsilon^{n}}\left[w\left(z, \tau_{o}\right)\right] d z & \geq \int_{K_{8}} \zeta^{p} \tau_{o} \chi_{\left[w\left(\cdot, \tau_{o}\right)<\epsilon^{n+1}\right]} d z \int_{0}^{1-\epsilon} \frac{\epsilon^{n(2-p)}}{[1+\epsilon-s]^{p-1}} d s \\
& \geq\left(Y_{n+1}-\varepsilon\right) \int_{0}^{1-\epsilon} \frac{\epsilon^{n(2-p)}}{[1+\epsilon-s]^{p-1}} d s .
\end{aligned}
$$

This and (B.25) yield

$$
Y_{n+1}-\varepsilon \leq Y_{n}[1-f(\epsilon)] .
$$

Estimate $f(\epsilon)$ below as follows. Let $\sigma_{1} \geq \sigma_{o}$ be defined by

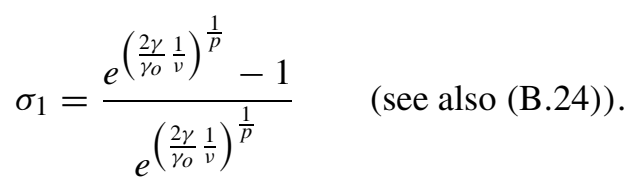

Then integrating the second integral on the right hand side of (B.26) over the smaller interval $\left[\sigma_{1}(1+\epsilon), 1\right]$, gives

$$
f(\epsilon)>\frac{1}{2}\left(1-\sigma_{1}\right)^{2-p}-\left(\frac{2 \epsilon}{1+\epsilon}\right)^{2-p} .
$$


Choose $\epsilon$ so small that

$$
f(\epsilon)>\frac{1}{4}\left(1-\sigma_{1}\right)^{2-p} \quad \text { and set } \quad \sigma=1-\frac{1}{4}\left(1-\sigma_{1}\right)^{2-p} .
$$

Since $\varepsilon \in\left(0, \frac{1}{4} \nu\right)$ is arbitrary, $Y_{n+1} \leq \sigma Y_{n}$, thereby establishing the proposition under the assumption that (B.10) holds true.

\section{B.7. Removing the assumption (B.10)}

Inequality (B.16) holds in any case in the integrated form

$$
\begin{aligned}
\int_{K_{8}} \zeta^{p} \tau \Phi_{k}[w(z, \tau)] d z & -\int_{K_{8}} \zeta^{p}(\tau-h) \Phi_{k}[w(z, \tau-h)] d z \\
& +\gamma_{o} \int_{\tau-h}^{\tau} \int_{K_{8}} \zeta^{p} s \Phi_{k}[w(z, s)] d z d s \leq \gamma h .
\end{aligned}
$$

Divide by $h$ and let $h \rightarrow 0$ to obtain (B.16) where the term involving the $\tau$ derivative is replaced by

$$
\left(\frac{d}{d \tau}\right) \int_{K_{8}} \zeta^{p} \tau \Phi_{k}(w) d z=\limsup _{h \rightarrow 0} \frac{1}{h}\left\{\int_{K_{8}} \zeta^{p} \tau \Phi_{k}(w) d z-\int_{K_{8}} \zeta^{p}(\tau-h) \Phi_{k}(w) d z\right\} .
$$

Introduce the set

$$
S=\left\{\tau \in(0,1] \mid\left(\frac{d}{d \tau}\right)^{-} \int_{K_{8}} \zeta^{p} \tau \Phi_{k}(w) d z \geq 0\right\}
$$

and let $\tau_{o}$ be defined as in (B.19). If $\tau_{o} \in S$, then

$$
\int_{K_{8}} \zeta^{p} \tau_{o} \Psi_{k}^{p}(w) d z \leq \gamma
$$

If $\tau_{o} \notin S$ but $\sup \left\{\tau<\tau_{o} \mid \tau \in S\right\}=\tau_{o}$, by working with a sequence of time levels $\tau_{n} \in S$ and $\left\{\tau_{n}\right\} \rightarrow \tau_{o}$, the previous inequality continues to hold. If $\tau_{o} \notin S$ and

$$
\tau_{*}=\sup \left\{\tau<\tau_{o} \mid \tau \in S\right\}<\tau_{o}
$$

we derive an inequality analogous to (B.22). The remainder of the proof remains the same. 


\section{References}

[1] Y. Z. Chen and E. DiBenedetto, Hölder estimates of solutions of singular parabolic equations with measurable coefficients, Arch. Ration. Mech. Anal. 118 (1992), 257-271.

[2] E. DE GiORGI, Sulla differenziabilità e l'analiticità delle estremali degli integrali multipli regolari, Mem. Accad. Sci. Torino. Cl. Sci. Fis. Mat. Nat. 3 (1957), 25-43.

[3] E. DiBENEDETTO, Intrinsic Harnack type inequalities for solutions of certain degenerate parabolic equations, Arch. Ration. Mech. Anal. 100 (1988), 129-147.

[4] E. DiBenedetto and Y. C. KWONG, Intrinsic Harnack estimates and extinction profile for certain singular parabolic equations, Trans. Amer. Math. Soc. 330 (1992), 783-811.

[5] E. DiBenedetto, Y. C. Kwong and V. Vespri, Local space analiticity of solutions of certain singular parabolic equations, Indiana Univ. Math. J. 40 (1991), 741-765.

[6] E. DiBenedetto, "Degenerate Parabolic Equations", Springer Verlag, Series Universitext, New York, 1993.

[7] E. DiBenedetto, U. Gianazza and V. Vespri, Harnack estimates for quasi-linear degenerate parabolic differential equations, Acta Math. 200 (2008), 181-209.

[8] J. HADAmARD, Extension à l'équation de la chaleur d'un théorème de A. Harnack, Rend. Circ. Mat. Palermo 3 (1954), 337-346.

[9] B. H. GILDING, On a class of similarity solutions of the porous media equation III, J. Math. Anal. Appl. 77 (1980), 381-402.

[10] J. Moser, On Harnack's theorem for elliptic differential equations, Comm. Pure Appl. Math. 14 (1961), 577-591.

[11] J. Moser, A Harnack inequality for parabolic differential equations, Comm. Pure Appl. Math. 17 (1964), 101-134.

[12] J. Moser, On a pointwise estimate for parabolic differential equations, Comm. Pure Appl. Math. 24 (1971), 727-740.

[13] M. A. PeletiER and H. ZHAng, Self-similar solutions of a fast diffusion equation that do not conserve mass, Differential Integral Equations 8 (1995), 2045-2064.

[14] B. PINI, Sulla soluzione generalizzata di Wiener per il primo problema di valori al contorno nel caso parabolico, Rend. Sem. Mat. Univ. Padova 23 (1954), 422-434.

Department of Mathematics

Vanderbilt University

1326 Stevenson Center

Nashville TN 37240, USA

em.diben@vanderbilt.edu

Dipartimento di Matematica "F. Casorati" Università di Pavia

via Ferrata, 1

27100 Pavia, Italia

gianazza@imati.cnr.it

Dipartimento di Matematica "U. Dini"

Università di Firenze

viale Morgagni, 67/A

50134 Firenze, Italia

vespri@math.unifi.it 